\title{
Degradation Behavior of Electrospun PLA and PLA/CNT Nanofibres in Aqueous Environment
}

\author{
Anna Magiera, ${ }^{1}$ Jaroslaw Markowski, ${ }^{2}$ Jan Pilch, ${ }^{2}$ and Stanislaw Blazewicz $\mathbb{}^{3}$ \\ ${ }^{1}$ Department of Heat Engineering and Environment Protection, Faculty of Metals Engineering and Industrial Computer Science, \\ AGH University of Science and Technology, 30 Mickiewicza Avenue, 30-059 Krakow, Poland \\ ${ }^{2}$ Laryngology Department, School of Medicine, Medical University of Silesia, 20 Francuska Str., 40-752 Katowice, Poland \\ ${ }^{3}$ Department of Biomaterials, Faculty of Materials Science and Ceramics, AGH University of Science and Technology, \\ 30 Mickiewicza Avenue, 30-059 Krakow, Poland \\ Correspondence should be addressed to Stanislaw Blazewicz; blazew@agh.edu.pl
}

Received 29 November 2017; Revised 10 February 2018; Accepted 22 February 2018; Published 1 April 2018

Academic Editor: Silvia Licoccia

Copyright (C) 2018 Anna Magiera et al. This is an open access article distributed under the Creative Commons Attribution License, which permits unrestricted use, distribution, and reproduction in any medium, provided the original work is properly cited.

\begin{abstract}
The aim of the work was to compare the degradation behavior of electrospun nanofibres obtained from pure poly(lactic acid) (PLA) and modified with carbon nanotubes (CNTs) in aqueous environment. The nanofibres in the form of mats were manufactured using the electrospinning technique (ES) with potential biomedical application. To investigate the degradation behavior, onecomponent and composite (containing CNTs) nanofibres were compared using scanning electron microscopy (SEM), water contact angle measurements, differential scanning calorimetry (DSC), and mechanical testing. The changes in their morphology, structure, and selected physical and mechanical properties during incubation up to 14 days were analysed. Two types of CNTs differing in concentration of surface functional groups were used to modify the PLA nanofibres. PLA and composite nanofibres (PLA + CNT) during incubation underwent swelling and partial degradation due to the penetration of water into polymer matrix. Changes in the mechanical properties of composite mats were higher than those observed for pure PLA mats. After 14-day incubation, samples retained from 47 to $78 \%$ of their initial tensile strength, higher for PLA samples. Morphological changes in pure PLA nanofibres were more dynamic than in composite nanofibres. No significant changes in crystallinity, wettability, and porosity of the samples occurred.
\end{abstract}

\section{Introduction}

Electrospinning is a versatile method of obtaining fibrous structures for the wide scope of applications, including analytical chemistry, environmental protection, or medicine [1-3]. Considering the range of fibres diameters (from several micrometers to nanometers [4]) obtained via ES, this technique has an increasing importance in the biomaterials and tissue engineering fields.

Bearing in mind potential applications of the fibres obtained via ES in regenerative medicine and tissue engineering, the choice of PLA as the biocompatible biomaterial [5] seems reasonable. Many PLA-based fibres including pure PLA fibres [6-8] and composite structures [9-11], obtained by sequential $[12,13]$ or blend ES $[14,15]$, were studied by research groups. Numerous authors in their works have proven the beneficial role of PLA structures in enhancing tissues formation and regenerative processes [16-18]. Considering the similarities between the fibres obtained via ES and the extracellular matrix (ECM), structures acquired by ES technique provide promising materials for tissue engineering scaffolds, to regenerate bone [19-21], cartilage [22-24], or neural tissue [25-27]. For these reasons fibrous structures are also widely investigated to elaborate scaffolds to regenerate bone tissue, in the case of small bone defects occurred from illnesses, injuries, or tumour resections [21].

Multiwalled carbon nanotubes (MWCNTs) have the ability to promote stem cells differentiation towards bone cells [28] and enhance bone formation [29-31]. They also are used to improve mechanical properties of PLA fibres [3234]. Carbon nanotubes were proven to enhance electrical properties of the fibrous structures obtained via ES [32-34]. 
This property is important when stimulating bone tissue formation [35]. Considering the use of carbon nanotubes their toxicity should be mentioned as well. Many authors have tackled this problem suggesting negative influence on cell culture and living organisms [36-38], though an agreement was not achieved in this matter. What should be noted is the fact that numerous researchers claim that functionalised MWCNTs are less toxic and only slightly affect cells viability [39-41].

Bioresorbable polyhydroxyacids based implants seem to be a promising alternative to allogenic bone grafts. Such bioresorbable implants can be manufactured from pure polymers and/or polymers/ceramic composites. While the degradation mechanism of polyhydroxyacids-derived nonporous and porous materials designed for scaffolds with different chemical composition is well recognised in the literature, much less is known about nanofibres, and, in particular, the effect of nanocomponents including CNTs on degradation of composite nanofibres in living organism. The fibrous polymeric materials, like the ones presented in our work, have high specific surface area and low apparent density; hence they should undergo surface degradation. The analysis of papers related to nanofibrous biomaterials for tissue engineering applications indicates that several problems including degradation mechanism and relationship between mechanical and physical properties and degradation time are not yet fully recognised $[42,43]$. Electrospun PLAderived nanofibres in the form of mats have high surface-tovolume ratio and high porosity, and the scaffolds made of such nanofibres degrade differently than bulk PLA-derived implant. The degradation behavior is one of the crucial factors when considering using such scaffolds in tissue engineering $[44,45]$. The $\mathrm{pH}$ and temperature have a distinct effect on the rate of degradation. It was shown that intramolecular transesterification was the pathway for the degradation of the PLA-based fibrous products in basic, aqueous solution, whereas in acidic environment this process is slower [46]. The temperature distinctly accelerates the rate of degradation. For the PGA matrix, a significant increase in the crystallinity during the early stage was detected, as well as a gradual decrease during the later period, and this indicated that preferential hydrolytic degradation in the amorphous regions occurred with cleavage-induced crystallization, followed by further degradation in the crystalline region [47]. Biodegradation of electrospun PLA-based fibrous scaffolds in vitro was studied in the work [48]. It was shown that cell culture accelerated the nanofibrous scaffold degradation to a limited extent.

The purpose of the work was to evaluate changes in electrospun nanofibres made of pure PLA and PLA modified with MWCNTs during hydrolytic degradation at $37^{\circ} \mathrm{C}$ in deionized water. Our aim was to compare nanofibres in the form of mats (nonwovens) during degradation. All the fibrous samples were studied to characterise their morphology and evaluate physicochemical and mechanical properties. Incubation experiments were aimed at the determination of possible changes in materials morphology and their selected properties resulting from 14-day incubation in water.

\section{Materials and Methods}

2.1. Preparation of the Samples. In order to manufacture nanofibres, PLA (Ingeo ${ }^{\mathrm{TM}}$ Biopolymer 3251D, NatureWorks LLC, USA; $M_{w} 90,000-120,000 \mathrm{~g} \mathrm{~mol}^{-1}$, polydispersity index $=1.79$ ) was used. The solution of PLA (15\% wt./v.) was prepared in dichloromethane, DCM (Avantor Performance Materials Poland SA, Poland), and N,N-dimethylformamide, DMF (Avantor Performance Materials Poland SA, Poland) (3:1 wt. ratio). Solution was homogenised on a magnetic stirrer $(50 \mathrm{rpm})$ and gently heated $\left(30-35^{\circ} \mathrm{C}\right)$ for $24 \mathrm{~h}$.

PLA solution was then modified with two types of multiwalled carbon nanotubes (CNTs) (Nanostructured and Amorphous Materials Inc., USA), in the concentration of $1 \%$ (wt.) with respect to the PLA content. The first type of CNTs contained surface hydroxyl groups (catalogue number 1249 YJF), labelled in the study as CNT-OH. The second type of CNTs (catalogue number 1213NMGS) in the form of suspension in the DMF $\left(0.03 \mathrm{~g} \mathrm{~mL}^{-1}\right)$, labelled as $\mathrm{f}$-CNT, was additionally functionalised in the composition of inorganic acids, according to the procedure described elsewhere [49]. This modification introduced carbonyl and carboxyl groups on the surface of the CNTs, enabling their stable dispersion in the DMF. After such a modification the concentration of oxygen containing groups of CNTs was $10.67 \%$ wt. The adequate amount of the nanofillers (in the solid or liquid form) was added into previously prepared PLA solutions and stirred under gentle heating for $24 \mathrm{~h}$. Prior to the use, the suspensions were homogenised in the ultrasonic washer Sonic-0.5 (80 W; Polsonic Sp.j., Poland) for an hour.

The ES process of the PLA, PLA + CNT-OH, and PLA $+\mathrm{f}$-CNT fibres was conducted under processing parameters established in our previous studies $[50,51]$. The parameters were as follows: voltage $12 \mathrm{kV}$, needle inner diameter $0.9 \mathrm{~mm}$, needle-to-collector distance $3 \mathrm{~cm}$, collector rotation speed $100 \mathrm{rpm}$, deposition time $30 \mathrm{~min}$, and flow rate $0.1 \mathrm{~mL} \mathrm{~h}^{-1}$. Electrospun fibres were collected on the rotating mandrel covered with aluminum foil, from which they were separated prior to further study. The obtained samples were $40 \pm 4 \mu \mathrm{m}$ thick.

2.2. Characterisation of the Samples. In order to examine the morphology of the nanofibres, the scanning electron microscope (SEM) (Nova NanoSEM 200; FEI Company, USA) was used. The SEM images were analysed using the ImageJ, version $1.50 \mathrm{~b}$ freeware program to determine fibres diameters. Using segmental method, overall porosity of the mats was determined. Surface chemical properties were estimated using water contact angle $(\theta)$ measurements (DSA10; Kruss, Germany) enabling to evaluate the wettability of the mats. Deionised water used in the experiments was prepared in PURELAB UHQ apparatus, Elga LabWater (USA). For each type of the sample, thirty individual measurements were made.

Mechanical testing of the fibrous mats was carried out on the universal testing machine Zwick 1435 (Zwick Roell, Germany) with load cell $5 \mathrm{kN}$. Samples in the form of strips $(4 \times 0.5 \mathrm{~cm})$ were placed between grips (distanced by $24 \mathrm{~mm}$ ) and elongated with the speed of $5 \mathrm{~mm} \mathrm{~min}^{-1}$. 


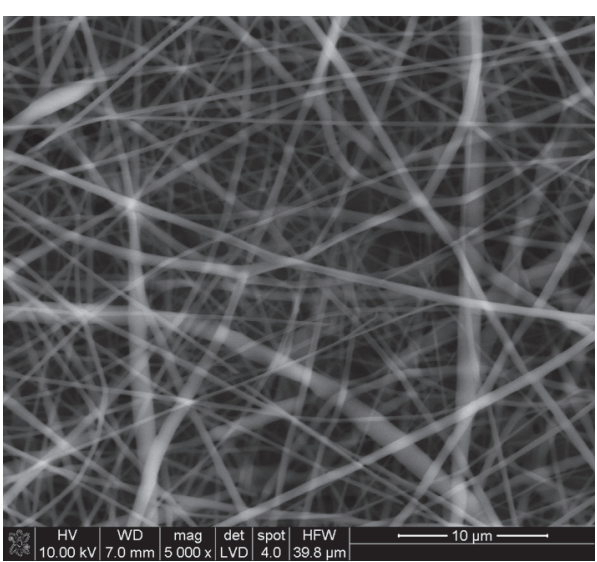

(a)

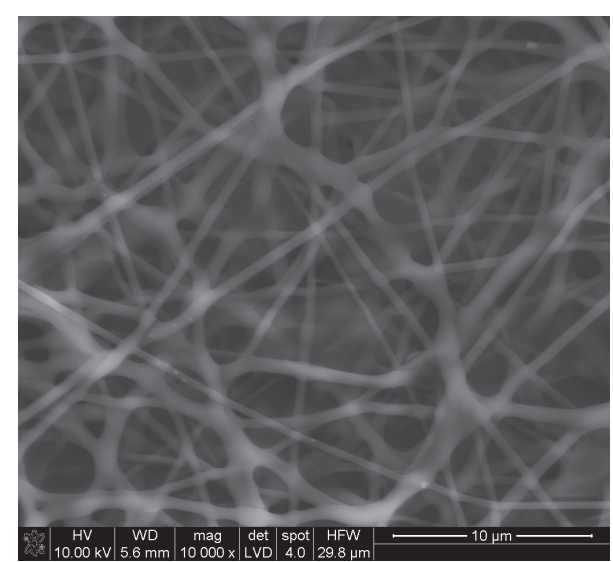

(b)

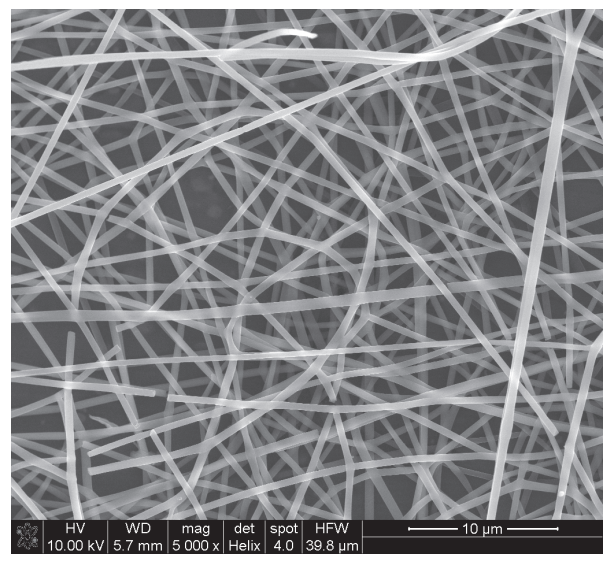

(c)

Figure 1: SEM microphotographs of nanofibres: (a) PLA, (b) PLA + CNT-OH, and (c) PLA + f-CNT.

Tensile strength, $R_{m}$, and Young's modulus, $E$, were calculated from the obtained stress-strain curves. Young's modulus was determined from the straight-line part of the tensile stressstrain function. For each type of the mats, three individual measurements were made.

Thermal analysis was carried out using differential scanning calorimeter (DSC) Mettler Toledo DSC1 (Mettler Toledo, Switzerland). The samples were analysed in the range of temperatures $0-200^{\circ} \mathrm{C}$ at $10^{\circ} \mathrm{C} \mathrm{min}^{-1}$ in air to determine the melting point $\left(T_{m}\right)$, the glass transition temperature $\left(T_{g}\right)$, the normalized heat of fusion $(h)$ of the PLA samples in the form of powder and nanofibres, and the onsets of phase transition during samples melting. The degree of crystallinity $\left(X_{c}\right)$ was measured assuming the enthalpy of fusion per gram for $100 \%$ crystalline PLA, $\Delta H_{m}$, to be $93 \mathrm{~J} \mathrm{~g}^{-1}$ [52]. The crystallisation percentage was calculated as the ratio of a fusion enthalpy measured from endothermic peak to the fusion enthalpy of $100 \%$ crystalline PLA.

2.3. Incubation Experiments. These experiments were performed in order to simulate degradation processes of the fibrous materials. Samples in the form of strips $(4 \times 0.5 \mathrm{~cm})$ were placed in containers filled with $30 \mathrm{~mL}$ of deionised water. Samples were then transferred to the mini incubator
Mini ICT 5.4 (Falc Instruments, Italy) and kept at $37^{\circ} \mathrm{C} \pm$ $0.5^{\circ} \mathrm{C}(\mathrm{pH}=5)$, for 3-14 days. Samples were then removed from the incubator and containers, rinsed twice with distilled water, and left for drying in a desiccator at room temperature, for one week. Dried materials were analysed using SEM, $\theta$ measurements, mechanical testing, and thermal analysis. For each incubation time mean values and standard deviation of three measurements were calculated.

\section{Results and Discussion}

SEM images of the fibrous mats acquired are presented in Figure 1. The overall porosity of all materials was very high, ranging from 0.88 to 0.91 (Table 1). Since biomaterials for tissue engineering purposes should have high porosities enabling cells infiltration during cells culture and after implantation [53], the data obtained are very favourable towards this application. The average diameter of pure PLA fibres is slightly higher compared to the diameters of PLA fibres modified with CNTs (Table 1). The addition of CNT$\mathrm{OH}$ or $\mathrm{f}-\mathrm{CNT}$ increased the electrical conductivity of the PLA solution, resulting in favourable jets splitting in the electrical field and smaller fibre diameters. Nonwovens were well formed, uniform, and defect-less. Because f-CNT were 
TABLE 1: Characterisation of mats.

\begin{tabular}{lcc}
\hline Sample & Fibre diameter, $\mathrm{nm}$ & Overall porosity \\
\hline PLA & $498 \pm 22$ & $0.91 \pm 0.01$ \\
PLA + CNT-OH & $456 \pm 30$ & $0.88 \pm 0.01$ \\
PLA + f-CNT & $306 \pm 17$ & $0.89 \pm 0.01$ \\
\hline
\end{tabular}

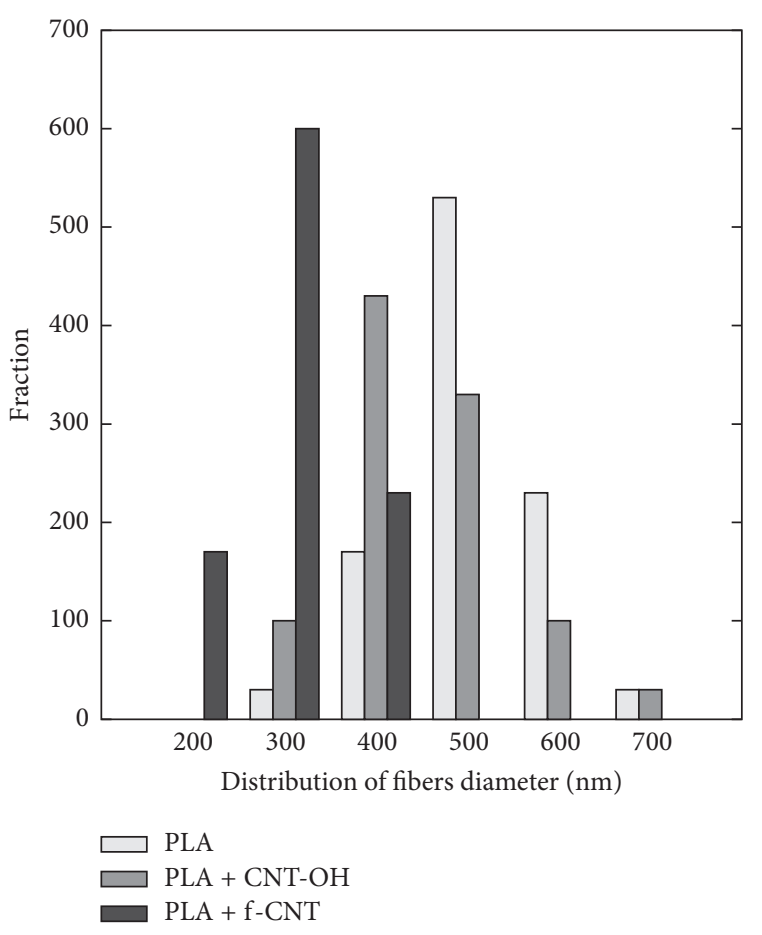

FIGURE 2: Distribution of nanofibres diameters in mats.

incorporated into the polymeric solution in the form of stable suspension in DMF (component of PLA solution), their dispersion was better than $\mathrm{CNT}-\mathrm{OH}$. As a result, PLA + f-CNT nanofibres were the thinnest of all materials analysed. Figure 2 presents the distribution of nanofibres diameters in PLA, PLA + CNT-OH, and PLA + f-CNT mats. Fibrous structures acquired were highly uniform; the ranges of the calculated fibres diameters were up to $10 \%$.

Water contact angle measurements were performed in order to evaluate chemical nature of the materials surfaces. The calculated value for PLA mat was about $113^{\circ}$ (Table 2), which is characteristic for hydrophobic materials $[15,54]$. PLA nanofibres modified with CNTs exhibit slightly smaller values, approximately $106-108^{\circ}$, which is probably related to the thinner polymeric fibres. $\theta$ values registered for two different modifiers were similar, because their general characteristics are comparable, and they differ only with respect to functional groups.

The mechanical tests revealed significant differences between the samples. Although the mechanical parameters of the porous mats are not too high compared to the typical values of PLA nanofibres, it is worth noting that the samples constitute highly porous materials (about 90\%). The tensile strength of these samples was determined from the tensile force-to-cross-section ratio of the porous samples (0.88-0.91,
Table 1). The nanofibres in mats were randomly oriented; therefore in the stretching test only a part of single nanofibres transferred the external load. For this reason, the actual strengths and modules of the individual nanofilaments are significantly higher. The recalculated mechanical parameters of the samples, taking into account the porosity (the crosssection area of the samples subjected to the tensile test is reduced) are given in Table 2 in brackets. As is apparent from this table the mechanical properties of nonporous samples are one order of magnitude greater than the values determined for the porous samples.

The pure PLA mats exhibited lower mechanical properties $\left(R_{m}=10.6 \mathrm{MPa}, E=686 \mathrm{MPa}\right)$ as compared to mats consisting of composite nanofibres. The addition of CNTs into PLA fibres improved their mechanical properties. Moreover, PLA + f-CNT fibres represent much better mechanical properties when compared to PLA $+\mathrm{CNT}-\mathrm{OH}$ and this observation results from better dispersion of $\mathrm{f}-\mathrm{CNT}$ in PLA matrix and, due to their chemically fuctionalised surface, from a stronger interaction influencing mechanical performance.

Our previous FTIR study on PLA and CNTs-modified PLA showed that due to a small concentration of CNTs in polymer nanofibre ( $1 \% \mathrm{wt}$.), no changes in the position of the bands characteristic for pure PLA were observed. A comparison between spectra of pure PLA and PLA + f-CNT showed that the bands assigned to PLA did not shift their wavenumbers in composite nanofibres [50].

DSC curves of the pristine polymer (PLA0) and PLA nonwovens are presented in Figure 3. Physical parameters of the samples calculated from DSC curves are collected in Table 3. In the first part of the PLA curve $\left(0-50^{\circ} \mathrm{C}\right)$ a slight endothermic effect was observed (Figure 3(a)) which was related to the evaporation of small amount of water, penetrating polymeric chains. In the case of pristine PLA0 polymer this effect was not observed. This difference originates from high specific surface area of the electrospun fibres when compared to original polymer form (granules) and the related higher moisture absorption. The degree of crystallinity calculated for PLA nonwoven was $45 \%$ ( $X_{c}$ for PLA0 was similar, $46 \%)$. Changes in materials structure in case of both PLA0 and PLA in the form of nanofibres were similar, with comparable corresponding $T_{m}$ and $h$ values.

Complete melting temperature was determined from the peak melting temperature of DSC curves. The curves obtained for PLA nonwovens modified with CNTs (Figure 3(b)) were less complex than the one measured for pristine PLA scaffold. Samples containing CNTs underwent only partial and complete melting; neither secondary crystallisation nor relaxation was observed. These transformations occurred at similar temperatures and exhibited similar specific enthalpy values (Table 3 ). The table shows that the values of the glass transition temperatures do not significantly vary between the samples and the modification of the PLA with CNT does not significantly affect this temperature. Three types of samples, that is, PLA precursor, pure PLA nanofibres, and nanofibres modified with $\mathrm{CNT}-\mathrm{OH}$, were characterised by comparable degree of crystallinity $X_{c}$, whereas higher crystallinity values were noted for nonwovens containing 
TABLE 2: Characterisation of fibrous mats; the values in brackets correspond to the properties of the nonporous samples.

\begin{tabular}{lccr}
\hline Sample & Wettability, $^{\circ}$ & Tensile strength, MPa & Young's modulus, MPa \\
\hline PLA & $113.3 \pm 1.0$ & $10.6 \pm 0.4(117.8)$ & $686 \pm 42(7622)$ \\
PLA + CNT-OH & $106.2 \pm 1.4$ & $12.2 \pm 0.5(101.7)$ & $634 \pm 17(5283)$ \\
PLA + f-CNT & $108.3 \pm 1.4$ & $16.3 \pm 2.0(147.2)$ & $1054 \pm 112(10582)$ \\
\hline
\end{tabular}

TABLE 3: Physical parameters of pristine (PLA0) and electrospun PLA calculated from DSC curves.

\begin{tabular}{|c|c|c|c|c|}
\hline Sample & Complete melting temperature, ${ }^{\circ} \mathrm{C}$ & Complete melting enthalpy, $\mathrm{J} \mathrm{g}^{-1}$ & Crystallinity, \% & Glass transition temperature, ${ }^{\circ} \mathrm{C}$ \\
\hline PLA0 & 171.60 & 42.78 & 46 & 68.97 \\
\hline PLA & 169.16 & 41.52 & 45 & 68.61 \\
\hline $\mathrm{PLA}+\mathrm{CNT}-\mathrm{OH}$ & 171.66 & 43.88 & 47 & 68.31 \\
\hline $\mathrm{PLA}+\mathrm{f}-\mathrm{CNT}$ & 169.94 & 47.03 & 51 & 70.45 \\
\hline
\end{tabular}

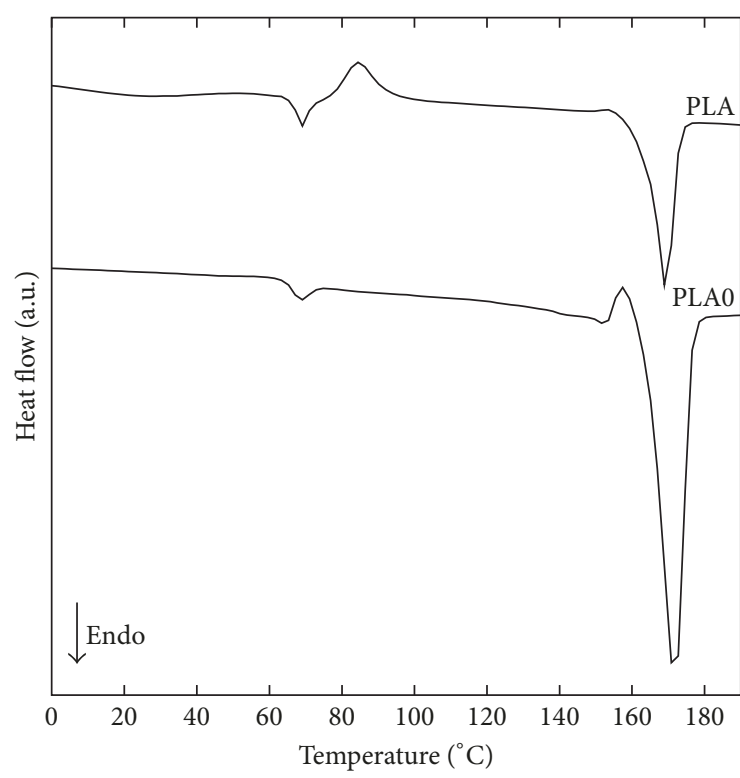

(a)

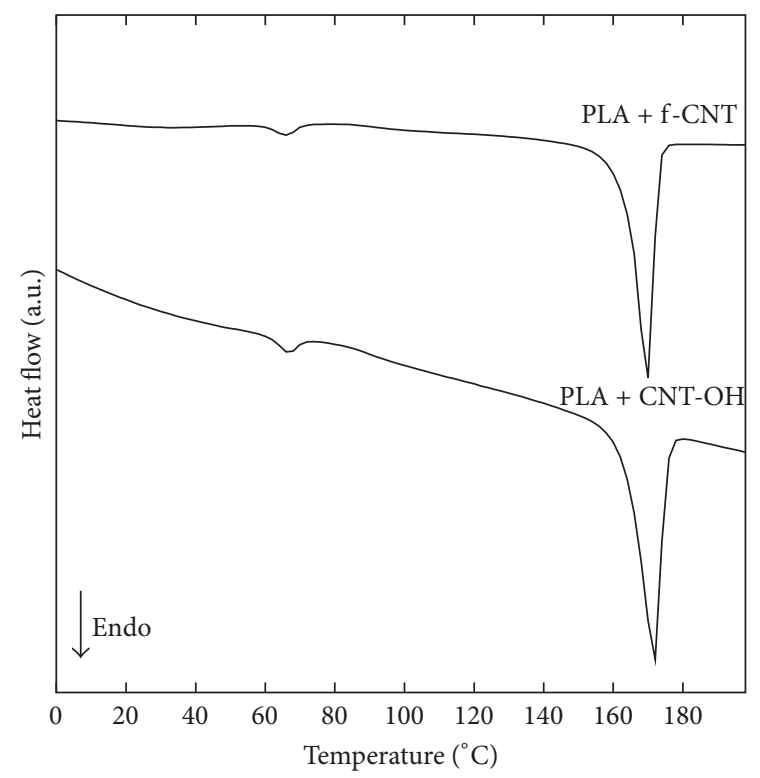

(b)

FIgURE 3: DSC curves of (a) pristine polymer (PLA0) and PLA mat and (b) PLA + CNT mats.

functionalised $\mathrm{f}-\mathrm{CNT}(51 \%)$. As is apparent from the mechanical tests despite a small content of f-CNT (1\%) in PLA nanofibres, significant improvement in mechanical properties and a reduction in the diameter of these fibres were achieved. Thus, the increase in crystallinity for PLA + f-CNT derived nonwovens may be explained by stronger interaction of $\mathrm{f}-\mathrm{CNT}$ with the polymer matrix during electrospinning. The CNTs surface may be a site for heterogeneous crystallisation during the polymer solidification process of electrospun fibres in the electric field. During ES process of PLA nanofibres, an evaporation of the solvent takes place and, depending on the ES conditions, the solvent rich sites may be transformed into nanofibre pores. In the case of thinner nanofibres, removal of the solvent occurs more easily; therefore it can be expected that the pores in these nanofibres are smaller in size. For these samples, slight endothermic effect in the range of $0-50^{\circ} \mathrm{C}$ related to water evaporation was observed as well. It can be thus concluded that the incorporation of $\mathrm{f}-\mathrm{CNT}$ in the fibrous polymer structure slightly affected thermal properties of the nonwovens.

The aim of the incubation experiments was to evaluate the degradation of electrospun polymeric structures in aqueous environment. Results of water contact angle measurements, mechanical testing, and DSC measurements are gathered in Figure 4 and Table 4. SEM microphotographs of mats after incubation are presented in Figure 5. It can be seen that when incubation time is prolonged, the primary fibrous structure begins to degrade. The well-formed, thin PLA fibres, assembled in a dense structure, underwent deformation and partial deterioration (Figures 5(a), 5(d), 5(g), and $5(\mathrm{j})$ ). The increase in nanofibres diameters indicates their swelling during incubation in water (Figure 4(a)). Figure 6 displays the evolution of nanofibres diameter distribution in mats during degradation. A progressive increase in the mean diameter of the samples can be seen, while maintaining the standard deviations on a similar level. At day 14 the increase 


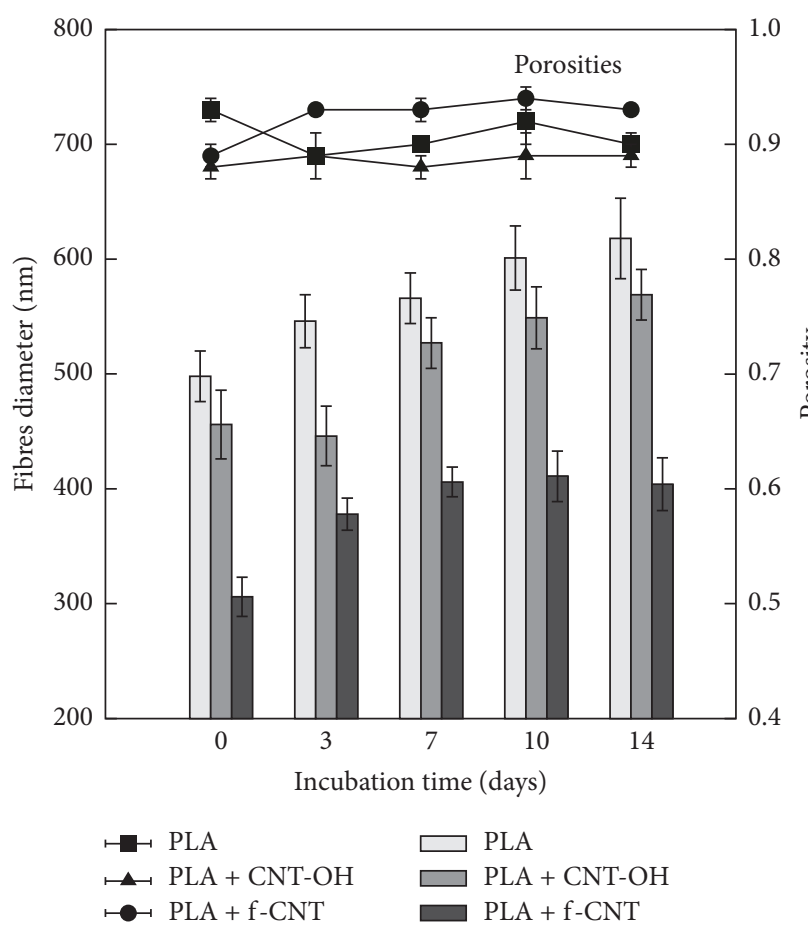

(a)

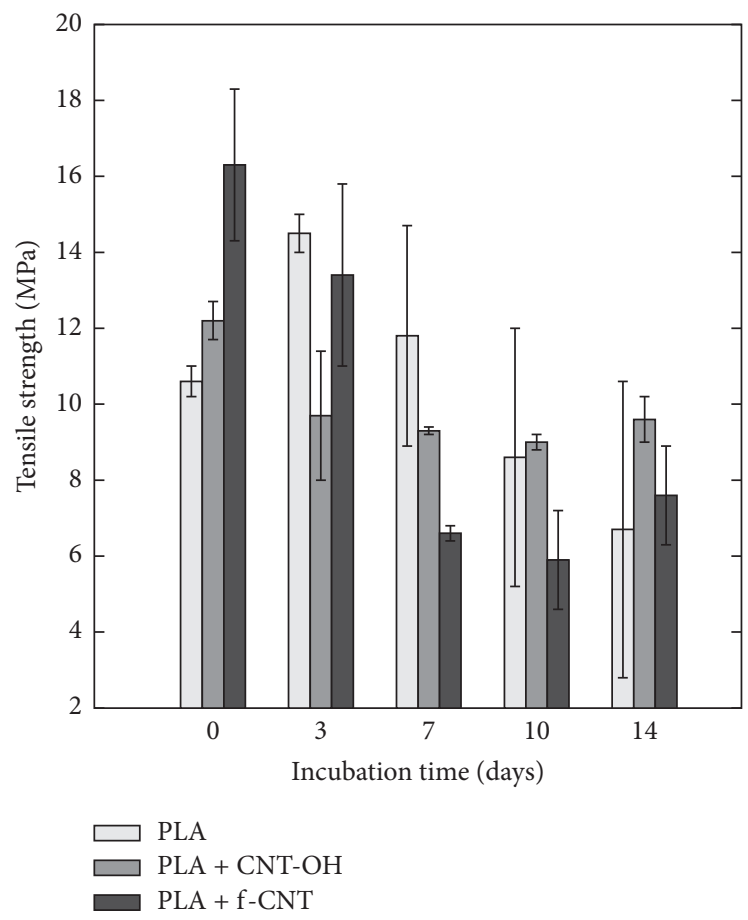

(c)

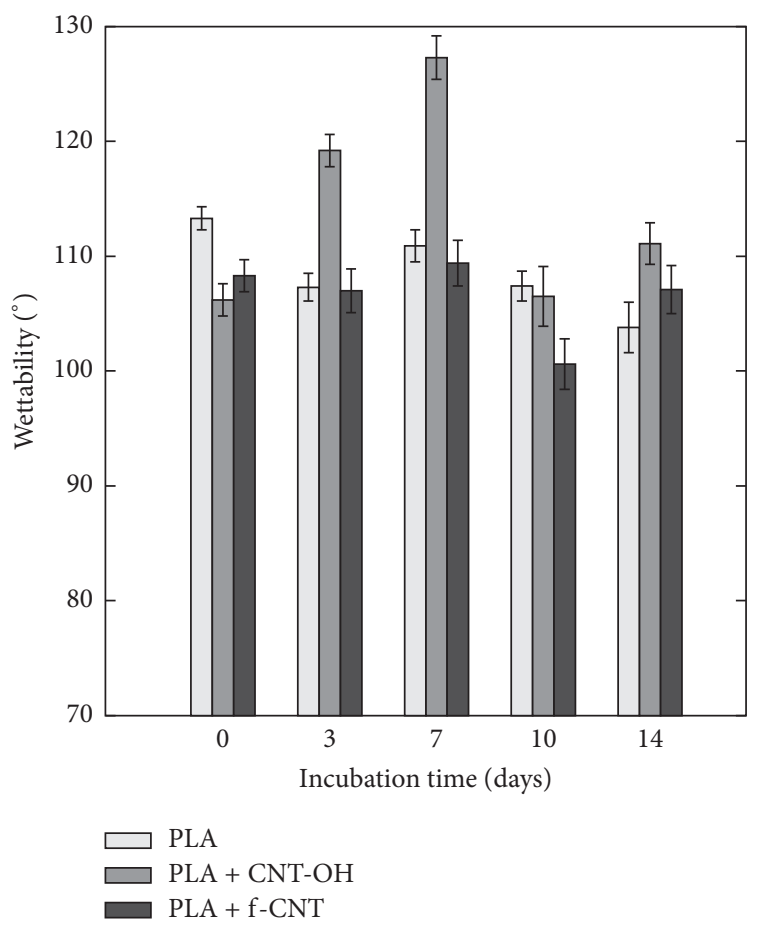

(b)

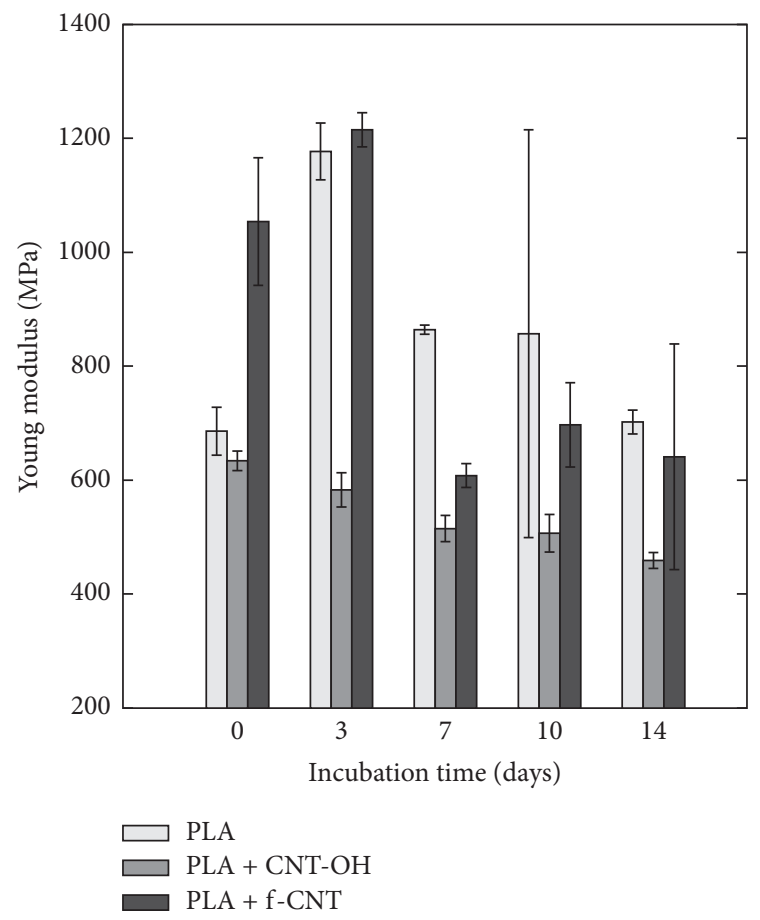

(d)

FIGURE 4: Characterisation of nanofibres in mats: (a) diameter/porosity, (b) wettability, (c) tensile strength, and (d) Young's modulus after incubation in water.

in the mean diameter of nanofibres was $12-13 \%$ for all types of mats.

PLA mats modified with CNTs after incubation are presented in Figures 5(b), 5(e), 5(h), 5(k), 5(c), 5(f), 5(i), and 5(l). Up to 2 weeks no degradation of fibrous structures was observed. Mats swelled and nanofibres merged due to prolonged incubation in water. The changes occurring in fibrous structure are analogous to those observed for pristine PLA samples, but less dynamic; no changes in nanofibres morphologies were evidenced. During the whole duration 
TABLE 4: Physical parameters of mats after incubation in water calculated from DSC curves.

\begin{tabular}{|c|c|c|c|c|c|c|}
\hline Sample & Incubation time & $\begin{array}{l}\text { Complete melting } \\
\text { temperature, }{ }^{\circ} \mathrm{C}\end{array}$ & $\begin{array}{c}\text { Complete melting } \\
\text { enthalpy, } \mathrm{Jg}^{-1}\end{array}$ & $\begin{array}{c}\text { Cold crystallization } \\
\text { enthalpy, } \mathrm{Jg}^{-1}\end{array}$ & Crystallinity, \% & $\begin{array}{l}\text { Glass transition } \\
\text { temperature, }{ }^{\circ} \mathrm{C} \\
\end{array}$ \\
\hline \multirow{4}{*}{ PLA } & 3 days & 169.12 & 45.39 & - & 49 & 69.78 \\
\hline & 7 days & 169.84 & 43.90 & - & 47 & 71.09 \\
\hline & 10 days & 169.90 & 47.62 & - & 51 & 71.79 \\
\hline & 14 days & 169.84 & 43.90 & - & 47 & 70.45 \\
\hline \multirow{4}{*}{$\mathrm{PLA}+\mathrm{CNT}-\mathrm{OH}$} & 3 days & 168.94 & 43.93 & 3.38 & 43 & 68.84 \\
\hline & 7 days & 169.32 & 42.37 & 3.41 & 42 & 67.96 \\
\hline & 10 days & 169.39 & 42.75 & 3.51 & 42 & 68.21 \\
\hline & 14 days & 169.42 & 43.21 & 2.11 & 44 & 68.34 \\
\hline \multirow{4}{*}{$\mathrm{PLA}+\mathrm{f}-\mathrm{CNT}$} & 3 days & 169.54 & 44.79 & - & 48 & 70.87 \\
\hline & 7 days & 169.85 & 45.88 & - & 49 & 71.13 \\
\hline & 10 days & 169.76 & 50.16 & - & 55 & 71.54 \\
\hline & 14 days & 169.90 & 50.13 & - & 54 & 70.67 \\
\hline
\end{tabular}

of the incubation, fibres modified with $\mathrm{f}-\mathrm{CNT}$ were thinner than their equivalents containing CNT-OH. It can also be noticed in Figures 5(j), 5(k), and 5(l) that on the 14th day of incubation some nanofibres in the mat were glued. Bulk porosity of both types of the materials remained unchanged (approx. 89 and 93\%, resp., for PLA + CNT-OH and PLA + f-CNT (Figure 4(a))). Measurements of mass changes of the samples made after 14 days of the incubation indicate a slight increase in comparison to the mass of the starting samples, in the range of 0.5 to $1.5 \%$. This effect can probably be attributed to adsorbed water molecules that remained after the drying process (room temperature, one week).

As a result of the wettability studies, it has been observed that the contact angles values vary between $107^{\circ}$ and $125^{\circ}$, depending on the type of mat. The initial wettability of all the samples tested is similar, while during the incubation it slightly altered; the highest one was noted for PLA-CNT $+\mathrm{OH}$. These changes are about $10-15^{\circ}$ and after 2-week incubation the wettability of all samples diminishes, but the samples still retain their hydrophobic properties. These slight changes can be attributed primarily to a change in surface topography that is related to nanofibres diameters and porosity. As Figure 4(a) shows, the sample porosities do not change significantly during incubation; hence the only parameter associated with the sample surface is the mean diameter of the individual nanofibres that grows due to physical water absorption. The increase in diameter results in changes in surface roughness of the sample, which can explain slight changes in the contact angle. After partial degradation of the nanofibres, the water molecules penetrate into the interior, which is accompanied by the swelling process. The water contact angle is related to the surface roughness and thicker nanofibres form a more wetting surface.

Mechanical testing showed that when the incubation is prolonged, mechanical parameters $R_{m}$ and $E$ decrease (Figures 4(c) and 4(d)). The mechanical changes caused by hydrolytic degradation of mats can be observed in changes of their stress-strain characteristics. Examples of these changes are illustrated in Figure 7. In order to compare the shape of these curves, the stress-strain functions were plotted in a normalized $x-y$ system, performing relative stress and strain values. Mats form a layered structure consisting of chaotically oriented fibres without any preferred orientation. The pure PLA mats during stretching test up to 10 days of incubation show almost a straight line of mechanical characteristic, while after 14 days the relationship becomes nonlinear and the samples behave in a ductile manner. The observed decrease in fibre strength (Figure 4(c)) can be explained by its swelling due to the penetration of water into the polymer matrix. Water molecules inside the polymeric matrix may act as the plasticising phase in nanofibre responsible for the changes in its characteristic during stretching from almost linear to ductile one in the final phase, before failure. Interestingly, PLA + CNT-OH nanofibres show nonlinear mechanical characteristic upon all the incubation time. The ductile nature of the changes for the initial nanofibres and after 14-day incubation upon loading is probably due to the presence of CNT-OH, which are poorly bonded with the polymer structure. Due to small concentration of functional groups the CNT-OH are located between the polymeric chains and form weak crosslinking bonds. It is noteworthy that these nanofibres are characterised by the lowest value of Young's modulus. The modification of as received PLA nanofibres with CNT-OH caused the decrease in their modulus when compared to pure PLA and PLA + f-CNT nanofibres.

The pattern of degradation of PLA + f-CNT differs as compared to both of the other nanofibres (PLA, PLA + $\mathrm{CNT}-\mathrm{OH}$ ). Initially and after 14-day incubation they retain almost rectilinear characteristics, typical of brittle materials. The presence of functionalised f-CNT significantly increased their strength and modulus resulting from the formation of strong crosslinking bonds in the polymer matrix compared to PLA nanofibres. However, during the degradation, these nanofibres swell and, like other nanofibres, a marked reduction in mechanical parameters is observed. However, due to the strong bonds of the f-CNT with the PLA matrix, the observed decrease in mechanical parameters for these mats is significantly higher; the mats retained $47 \%$ of their 


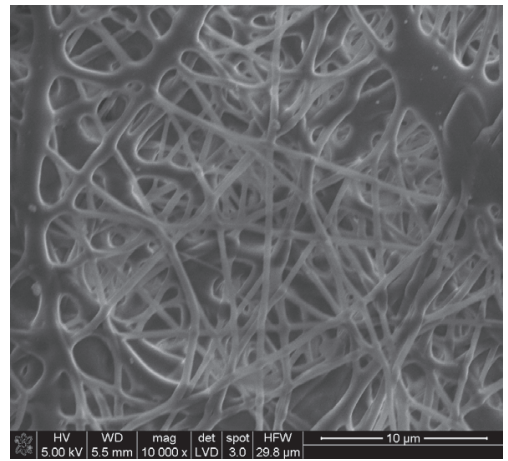

(a)

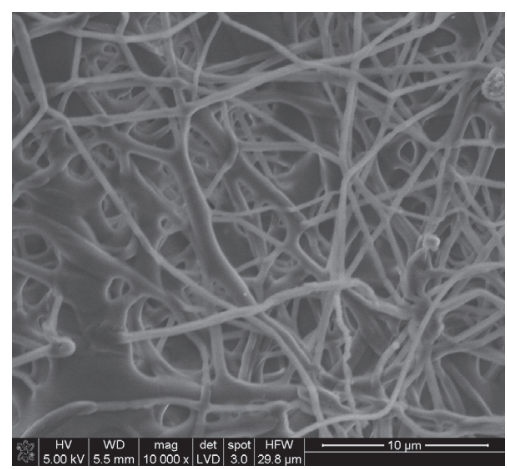

(d)

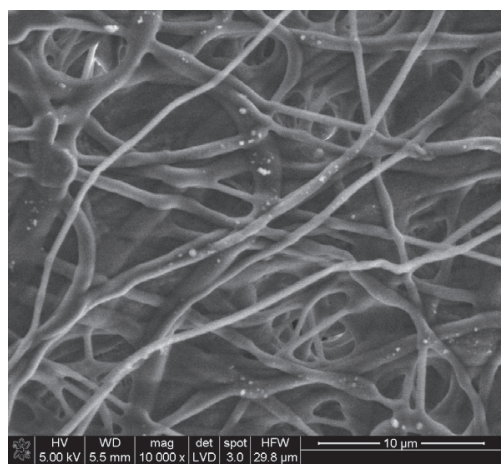

(g)

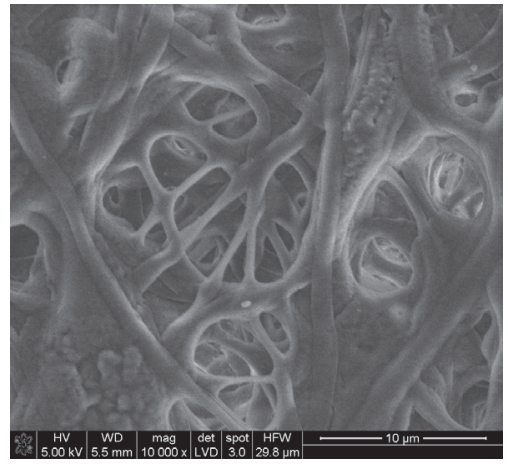

(j)

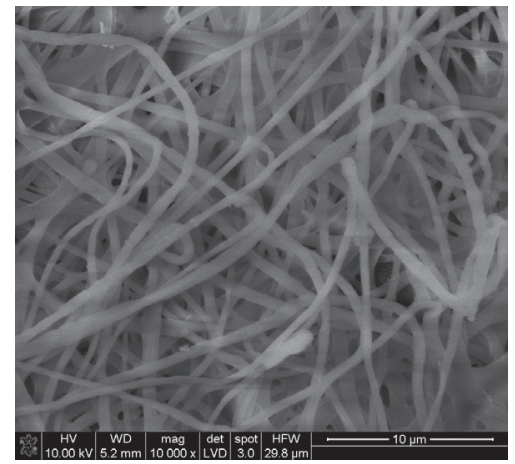

(b)

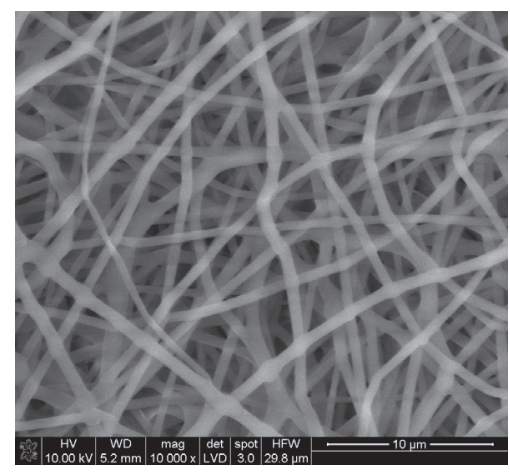

(e)

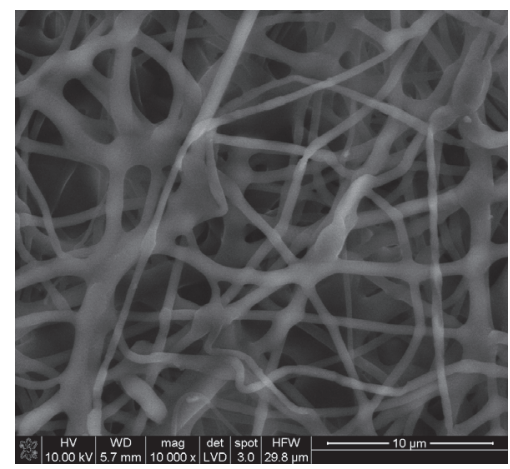

(h)

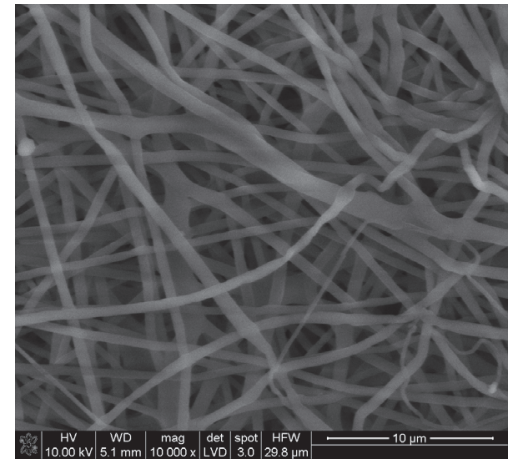

(k)

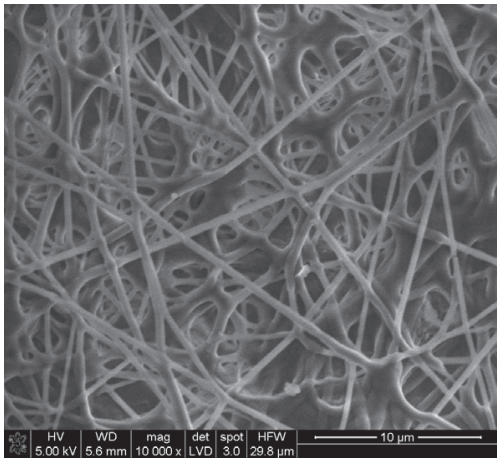

(c)

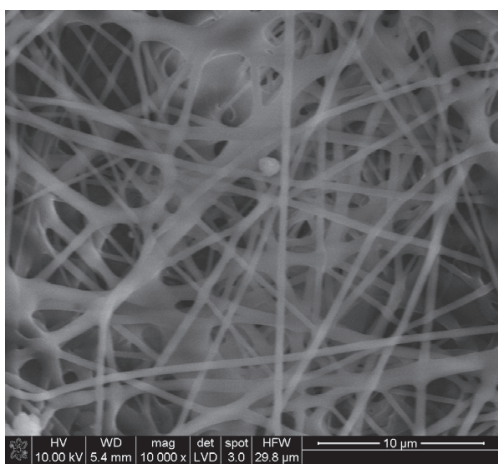

(f)

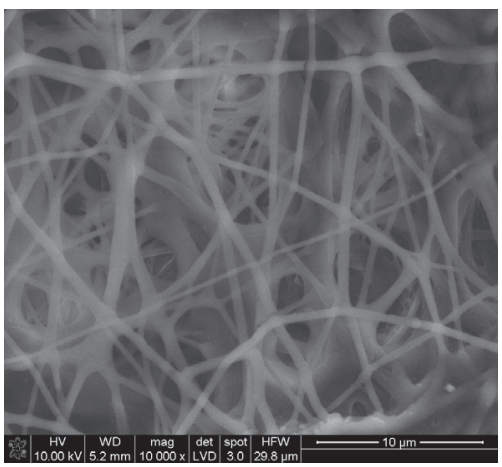

(i)

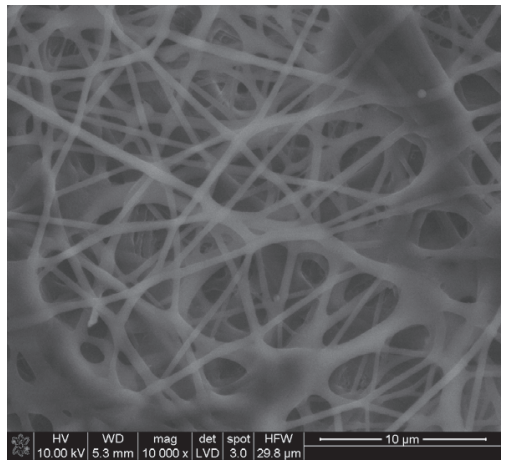

(l)

Figure 5: SEM microphotographs of mats: PLA ((a), (d), (g), and (j)), PLA + CNT-OH ((b), (e), (h), and (k)), and PLA + f-CNT ((c), (f), (i), and (l)), after $3((\mathrm{a}),(\mathrm{b})$, and $(\mathrm{c})), 7((\mathrm{~d}),(\mathrm{e})$, and $(\mathrm{f})), 10((\mathrm{~g}),(\mathrm{h})$, and $(\mathrm{i}))$, and $14((\mathrm{j}),(\mathrm{k})$, and $(\mathrm{l}))$ days of incubation in water. 


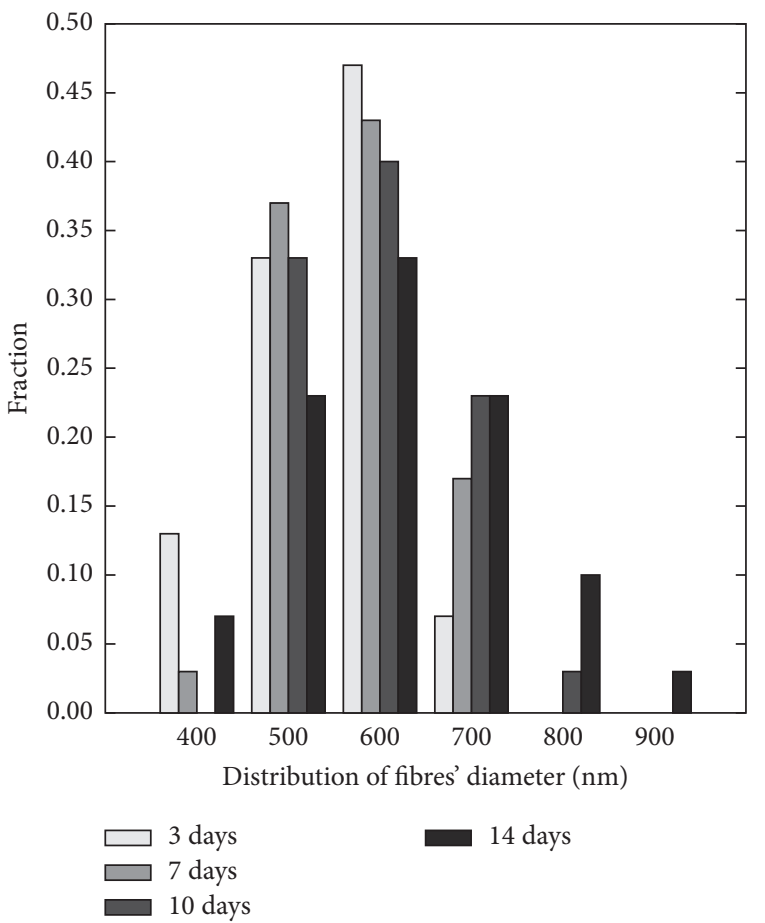

(a)

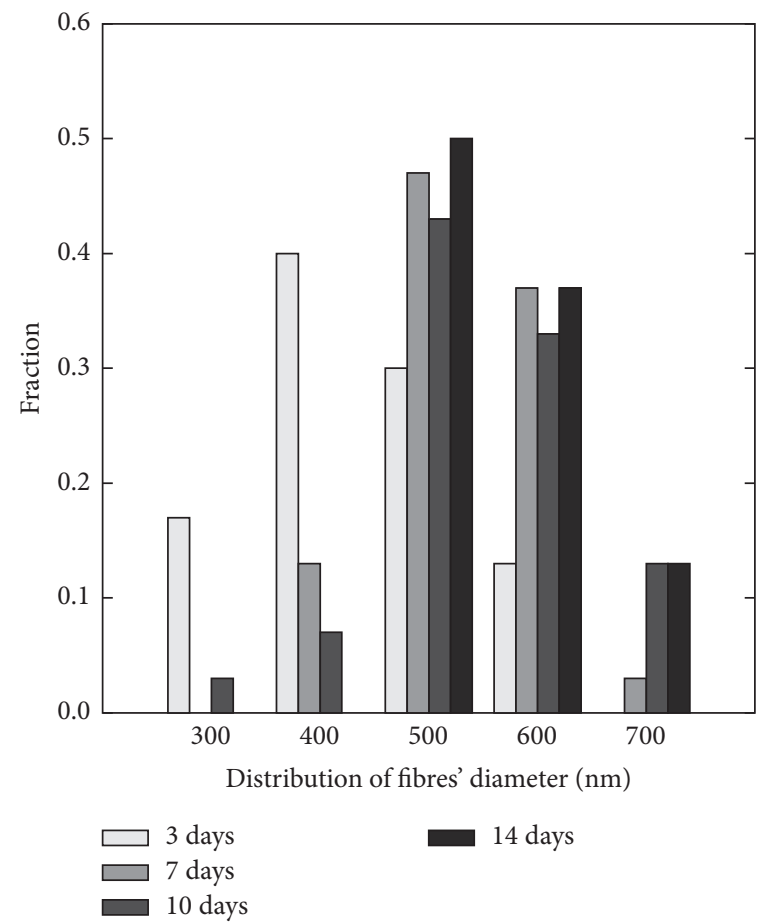

(b)

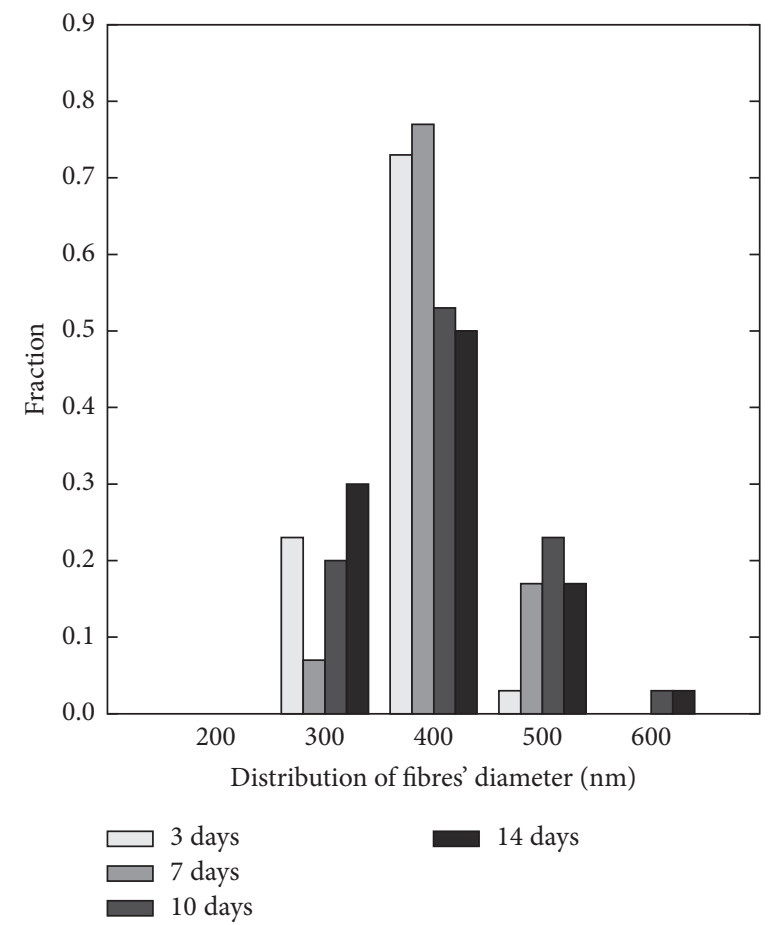

(c)

FIgURE 6: Distribution of nanofibres diameters in mats: (a) PLA, (b) PLA + CNT-OH, and (c) PLA + f-CNT after incubation in water.

initial strength, whereas the PLA and PLA + CNT-OH mats retained $63 \%$ and $78 \%$ of their initial strength, respectively. The nature of these changes indicates that the nanofibres forming the mats physically interact with the water that penetrates into pores of electrospun nanofibres and causes the increase in their diameter. In the case of PLA $+\mathrm{f}-\mathrm{CNT}$ the degradation has also chemical impact leading to the destruction of CNTs crosslinking bond with the polymer matrix. Prolonging the incubation time in water causes a decrease in tensile strength and modulus of both pure PLA 


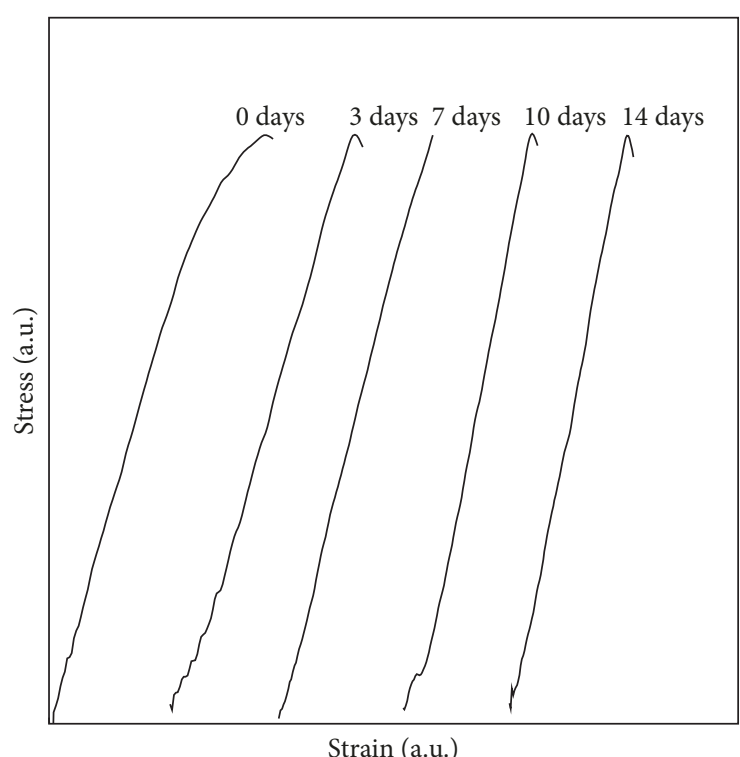

(a)

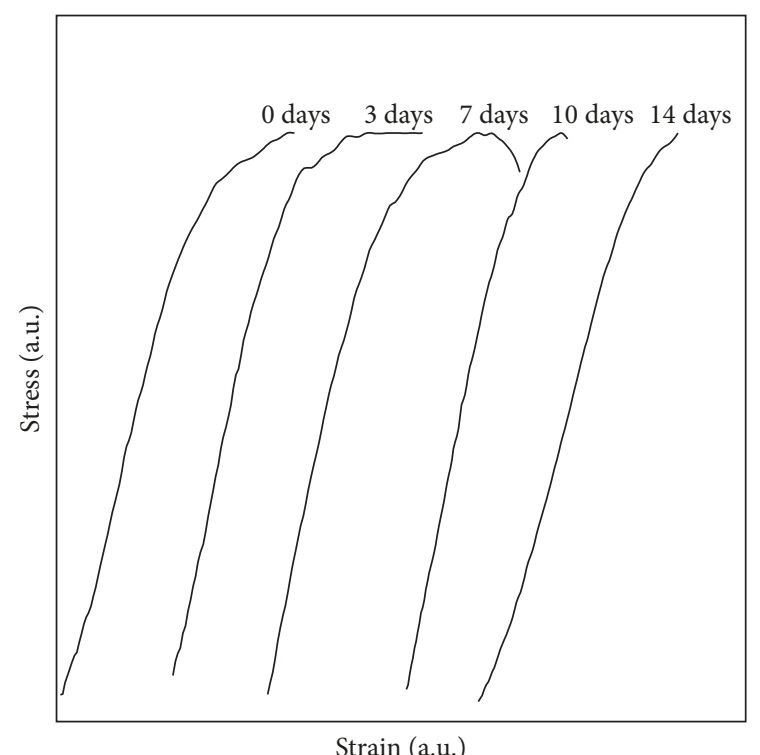

(b)

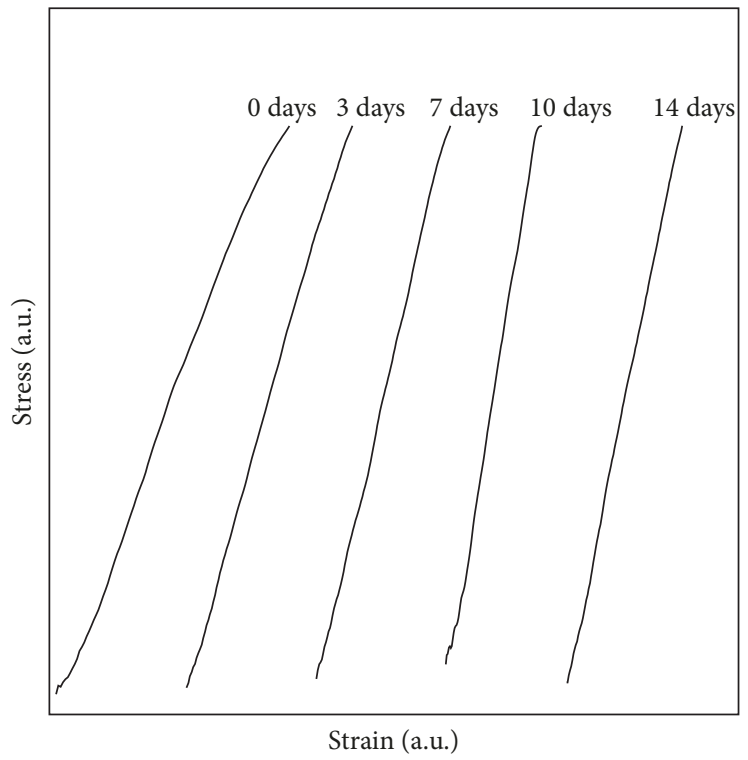

(c)

FIgURE 7: Stress-strain curves of (a) PLA, (b) PLA + CNT-OH, and (c) PLA + f-CNT mats after incubation in water.

and composite mats (Figures $4(\mathrm{c})$ and $4(\mathrm{~d})$ ). The reduction in mechanical parameters is accompanied by a marked increase in standard deviations.

The elongation changes (strains at break) of the samples show a complex picture of the processes occurring during incubation in water (Figure 8). These curves indicate that the strains at break for the samples decrease to the 10th day of incubation. This may be related to the observed swelling of the individual nanofibres and mainly applies to the pure PLA and PLA + f-CNT mats. On the contrary, PLA + CNT-OH mats behave differently; the samples are characterised by distinctly greater strain at break, which is also confirmed by the diagrams in Figure 7(b). After 14 days of the incubation, the increase in relative strains during the tensile test is observed, which may be explained by partial merging of individual nanofibres (Figures 5(j), 5(k), and 5(l)) and indicate the initial hydrolytic degradation process.

DSC curves of pure PLA mats after incubation are presented in Figure 9. In the first part of all the curves $\left(0-50^{\circ} \mathrm{C}\right)$ a slight endothermic effect was observed indicating the evaporation of small amount of water. The analysed materials underwent complete melting at about $169^{\circ} \mathrm{C}$ (Table 4). The specific enthalpy $h$ values of these processes were similar, as well as the crystallinity $X_{c}$ (approx. 50\%). In the case of incubated samples secondary crystallisation, observed during heating of pristine PLA mats (Figure 9(a)), was not detected. Such a difference can be explained by the presence of water in fibrous polymeric structure, which due to its 


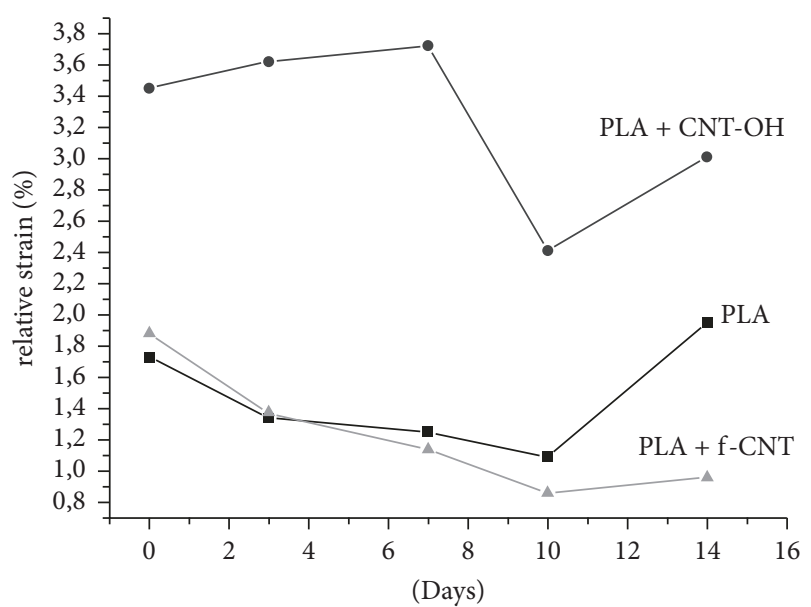

FIGURE 8: Variations of strain at break of pure PLA mat and modified with $\mathrm{CNT}$ as a function of incubation in water.

low thermal conductivity impedes the crystallisation. The calculated $h$ values for partial melting process are much lower compared to PLA before incubation, which implies that the polymeric chains have shortened during incubation.

DSC curves of PLA + CNT mats after incubation are presented in Figures 9(b) and 9(c). In the first part of all the curves $\left(0-50^{\circ} \mathrm{C}\right)$ an endothermic effect was observed indicating the evaporation of small amount of water. The analysed PLA + CNT-OH materials underwent secondary crystallisation and complete melting. They took place at temperatures, respectively, about $85^{\circ} \mathrm{C}$ and $169^{\circ} \mathrm{C}$ (Table 4). The specific enthalpy $h$ values of these processes were similar, as well as the crystallinity $X_{c}$ (approx. $42-44 \%$ ). It can be concluded that, during incubation, no significant changes at molecular level occurred; furthermore no advanced degradation of the materials was observed. The PLA + f-CNT mats underwent a partial and complete melting. The temperatures at which these processes occurred slightly increased as incubation time extended. An augmentation in samples crystallinity $X_{c}$ from about 48 to $54 \%$ explains an increase in melting temperatures and decrease in mechanical properties. This increase in crystallinity results from a partial degradation, which proceeds initially in the amorphous sites of the polymer, which are more accessible to water than the crystalline sites. Such a process usually leads to an increase of the sample crystallinity. However, the observed crystallinity changes are small and may indicate that the hydrolytic degradation of fibrous samples is slowed down. According to DSC analysis, slight changes in the values of $\mathrm{Tg}$ collected in Tables 3 and 4 upon incubation within 14 days were observed. It is known that hydrolytic degradation of PLA-based structure usually leads to a decrease in the $T g$ values that results in an increase in the mobility of the polymer chains due to a plasticizing effect [55]. Slight changes in the $\mathrm{Tg}$ values observed in our study probably result from a relatively short incubation time, when only fibre swelling was observed.

The times to complete degradation of PLA-based biomaterials in aqueous conditions depend on numerous factors including the form of the biomaterial itself (bulk, porous, fibrous, and powder), its molecular weight, polydispersity, crystallinity, surface energy, additives, and microstructure. Typical PLA biomaterials with low specific surface area (bulk materials) undergo degradation in volume and their molecular mass decreases due to the contact with water. Acidic degradation products of the material, which are released in aqueous and biological environments, accelerate the degradation process $[42,43,56,57]$. PLA mats, incubated in water, have a high specific surface area and relatively high molecular weight; it can be thus expected that they will hydrolytically degrade and the process will begin on their surface and in the open porous matrix of nanofibres. Degradation products may be easily removed due to high specific surface area and high open porosity of the samples, which diminishes the probability of autocatalytic hydrolysis effect $[42,43]$. In the case of mats studied in the work, at day 3 of degradation, small changes in nanofibres morphologies are already noticeable, by an increase in their diameters. The diameters of the nanofibres progressively increase up to 2 weeks of incubation (Figure 4(a)). Due to the presence of pores that were formed during rapid removal of the solvent from the ES process the penetration of water into the interior of the nanofibres takes place. Swelling and partial merging individual nanofibres were observed as a result of degradation of the samples. The hydrolytic reaction seems to be much slower, as evidenced by the lack of changes in the crystallinity (except for PLA + f-CNT), porosity, and wettability of the sample surface after incubation.

The addition of CNT-OH or f-CNT increased the electrical conductivity of the PLA solution, resulting in favourable jets splitting in the electrical field and smaller fibre diameters. Samples in the form of mats were well formed, uniform, and defect-less. Because f-CNT were incorporated into the polymeric solution in the form of stable suspension in DMF (component of PLA solution), their dispersion in PLA solution was better than CNT-OH. As a result, PLA $+\mathrm{f}-\mathrm{CNT}$ fibres were the thinnest of all materials analysed. Water contact angel measurements were performed in order to evaluate chemical nature of the materials surfaces. The calculated $\theta$ value for PLA mats was about $113^{\circ}$, which is characteristic for hydrophobic materials. PLA nanofibres modified with CNTs exhibit slightly smaller values, approximately $106-108^{\circ}$. $\theta$ values registered for two different modifiers were similar, because their general characteristics are comparable, and they differ only with respect to functional groups. DSC curves registered for pristine raw material and PLA mats, as well as $X_{c}$ and $T m$ values were similar, which indicates that polymer itself has not changed during ES process. The addition of CNTs is not clearly visible on DSC curves. That suggests the limited influence of the nanoadditive used on the thermal properties of the polymer. Mechanical testing revealed significant differences between analysed materials. PLA samples exhibited the lowest mechanical properties $\left(R_{m}=10.6 \mathrm{MPa}, E=686 \mathrm{MPa}\right)$. The addition of CNTs into PLA fibres improved their mechanical properties. For PLA + f-CNT fibres, the calculated values, $R_{m}=16.3 \mathrm{MPa}$ and $E=1054 \mathrm{MPa}$, were higher than the ones of PLA + CNT-OH $\left(R_{m}=12.2 \mathrm{MPa}, E=634 \mathrm{MPa}\right)$. This observation supports the conclusion of better dispersion of $\mathrm{f}-\mathrm{CNT}$ in PLA 


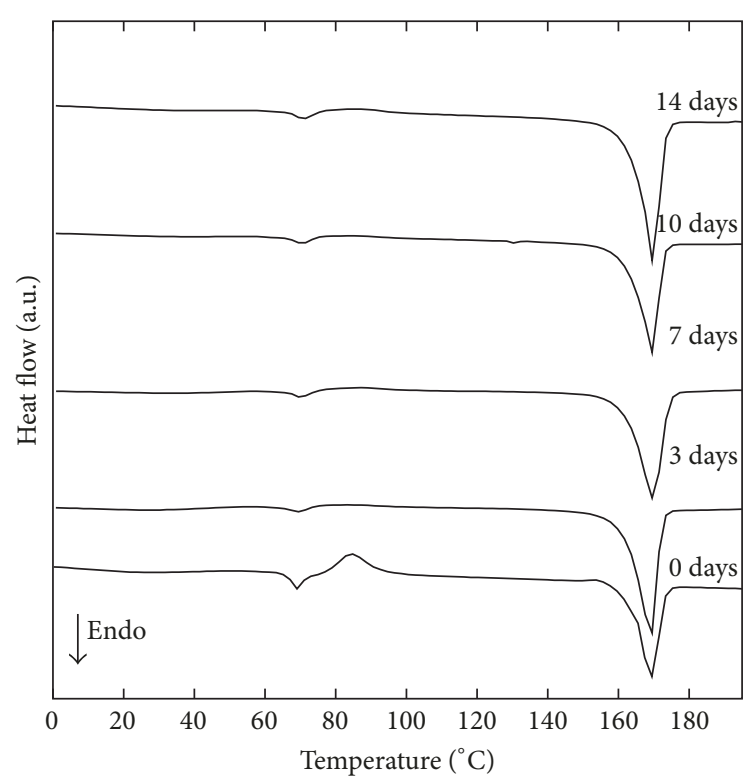

(a)

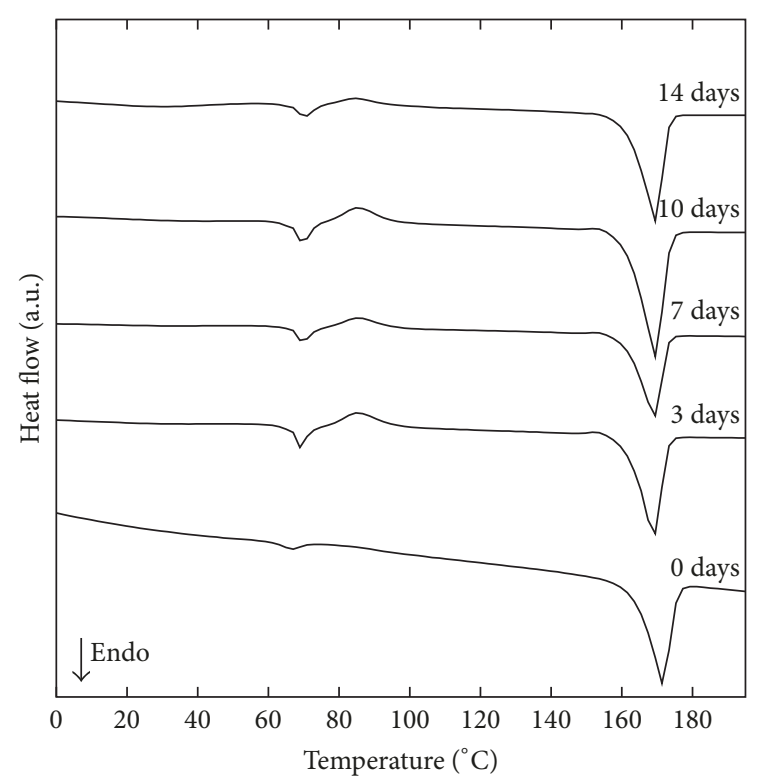

(b)

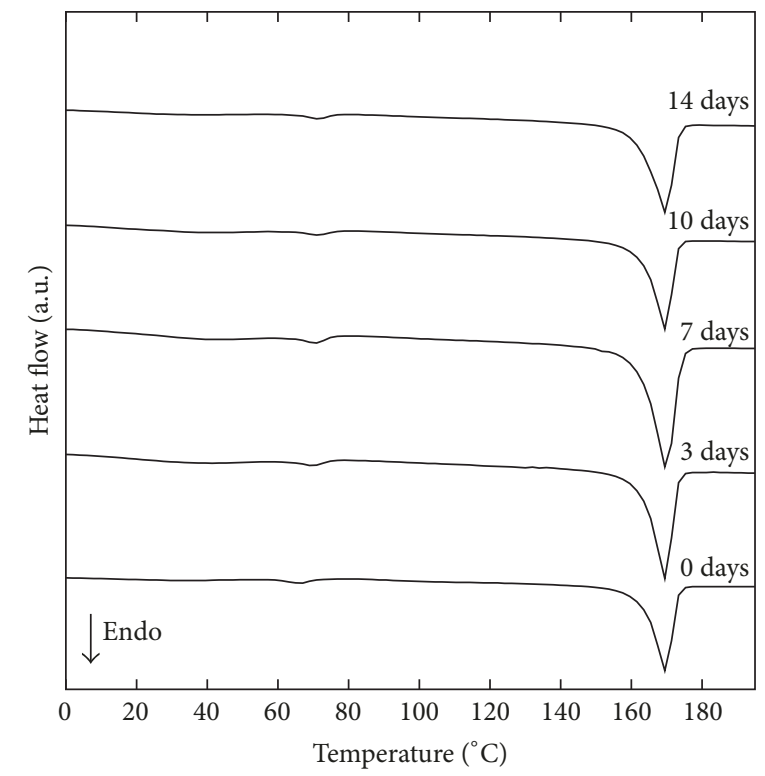

(c)

FIgURe 9: DSC curves of (a) PLA, (b) PLA + CNT-OH, and (c) PLA + f-CNT mats after incubation in water.

matrix, influencing its mechanical performance. Regarding low strength parameters and lack of mechanical handiness of PLA nonwovens, essential in case of biomaterials for tissue engineering scaffolds, the incorporation of CNTs within PLA matrix, described in this study, may be a way to overcome these difficulties. Another approach implies an increase in number of nanofibres per unit area of the material (surface density of mats), which would decrease the very high bulk porosity of these materials. Such an effect can be achieved by altering the ES process parameters, especially by decreasing collector velocity or aiming to obtain thinner nanofibres.

PLA and PLA + CNT materials underwent swelling and partial deformation of their fibrous structure. An increase of fibres diameters was observed, though the bulk porosity of the materials remained unchanged. Structural changes of PLA + CNT mats were less dynamic, which suggest their higher stability under the experiment conditions. Due to the high surface area of the nanofibres and high open porosity of the mats, their degradation process is physical in nature; that is, an increase of individual nanofibre diameter occurs, whereas near surface hydrolytic resorption results in a partial gluing the nanofibres. Degradation products originating from PLA matrix hydrolysis were easily removed, which decreased the rate of material decomposition in comparison to bulk implants degrading in volume. Water contact angle measurements showed slight changes in $\theta$ values, which implies lack 
of significant changes on the surface of the mats resulting from chemical properties or roughness changes. The calculated $R_{m}$ and $E$ values gradually decreased with incubation time. The standard enthalpy, $h$, value determined from DSC curves of PLA nonwovens diminished as well, which could issue from the shortening of the polymeric chains during incubation. Crystallinity, $X_{c}$, of the samples containing $\mathrm{f}-$ CNT was increasing. However, for these samples, a significant reduction in the mechanical properties was found. These mats have retained only $47 \%$ of the initial strength. This fact can be explained by the interaction of the aqueous environment at the interfacial phase formed between the CNTs functionalised surface and the polymer matrix. During the incubation experiment no changes at the molecular level of the materials were noted. Such a study should be repeated using more active medium, for example, PBS or SBF, and for longer incubation time.

\section{Conclusions}

The following conclusions can be drawn from the experimental work:

(1) Microstructural study of electrospun PLA-derived mats showed distinct differences between pure PLA samples and composite samples containing CNTs nanofillers; mats containing CNTs exhibited smaller diameters of single nanofibres.

(2) Wettability study of PLA mats indicated that pure PLA mats have water contact angles typical for hydrophobic materials, that is, about $113^{\circ}$. PLA nanofibres modified with CNTs exhibited slightly smaller contact angles, registered for both nanofillers (CNT-OH and $\mathrm{f}-\mathrm{CNTs}$ ).

(3) Tensile strength for composite nonwovens containing $1 \%$ wt. f-CNT increased from 10.6 MPa up to $16 \mathrm{MPa}$ while tensile modulus increased from $686 \mathrm{MPa}$ to $1050 \mathrm{MPa}$; this improvement is due to good dispersion of functionalised CNTs within PLA suspension. Additional functionalisation of the CNTs surface resulted in a significant decrease of nanofibres diameter. Improvements in the composites mechanical properties could also be attributed to better interphase attractive interaction and adhesion of the incorporated CNTs in the polymer matrix.

(4) Thermal studies performed using DSC technique for pristine PLA material and PLA mats indicated that polymer matrix has not changed its characteristics (crystallinity, $X_{c} T_{q}$ and $T_{m}$ values) during ES process. The effect of the addition of CNTs to polymer matrix was not visible on DSC curves.

(5) During 14-day incubation of fibrous structures in aqueous environment at $37^{\circ} \mathrm{C}$ all the samples underwent swelling and partial deformation of their integrity. An increase of fibres diameters was observed, whereas the overall porosity of the materials remained unchanged. Structural changes of PLA + CNT mats were less dynamic compared to pure fibrous samples, which suggest their higher stability under the experiment conditions. However, higher changes were noted in mechanical properties of composite samples with functionalised CNTs compared to pure samples, probably caused by hydrolytic degradation of PLA/CNTs interfacial bonding after 14 days of incubation.

\section{Conflicts of Interest}

The authors declare that there are no conflicts of interest regarding the publication of this article.

\section{Acknowledgments}

This work was supported by the National Science Center (Poland), Project no. 2013/11/N/ST8/01169.

\section{References}

[1] S. Chigome and N. Torto, "A review of opportunities for electrospun nanofibers in analytical chemistry," Analytica Chimica Acta, vol. 706, no. 1, pp. 25-36, 2011.

[2] R. Balamurugan, S. Sundarrajan, and S. Ramakrishna, "Recent trends in nanofibrous membranes and their suitability for air and water filtrations," Membranes, vol. 1, no. 3, pp. 232-248, 2011.

[3] L. Zhang, Y. He, G. Ma, C. Song, and H. Sun, "Paclitaxelloaded polymeric micelles based on poly( $\varepsilon$-caprolactone)poly(ethylene glycol)-poly( $\varepsilon$-caprolactone) triblock copolymers: in vitro and in vivo evaluation," Nanomedicine: Nanotechnology, Biology and Medicine, vol. 8, no. 6, pp. 925-934, 2012.

[4] A. Frenot and I. S. Chronakis, "Polymer nanofibers assembled by electrospinning," Current Opinion in Colloid \& Interface Science, vol. 8, no. 1-2, pp. 64-75, 2003.

[5] D. Garlotta, "A literature review of poly(lactic acid)," Journal of Polymers and the Environment, vol. 9, no. 2, pp. 63-84, 2001.

[6] X. Zong, K. Kim, D. Fang, S. Ran, B. S. Hsiao, and B. $\mathrm{Chu}$, "Structure and process relationship of electrospun bioabsorbable nanofiber membranes," Polymer Journal, vol. 43, no. 16, pp. 4403-4412, 2002.

[7] R. Inai, M. Kotaki, and S. Ramakrishna, "Structure and properties of electrospun PLLA single nanofibres," Nanotechnology, vol. 16, no. 2, pp. 208-213, 2005.

[8] L. D. Wright, R. T. Young, T. Andric, and J. W. Freeman, "Fabrication and mechanical characterization of 3D electrospun scaffolds for tissue engineering," Biomedical Materials, vol. 5, no. 5, Article ID 55006, 2010.

[9] N. D. Luong, I.-S. Moon, D. S. Lee, Y.-K. Lee, and J.-D. Nam, "Surface modification of poly(l-lactide) electrospun fibers with nanocrystal hydroxyapatite for engineered scaffold applications," Materials Science and Engineering C: Materials for Biological Applications, vol. 28, no. 8, pp. 1242-1249, 2008.

[10] J. B. Chiu, C. Liu, B. S. Hsiao, B. Chu, and M. Hadjiargyrou, "Functionalization of poly(L-lactide) nanofibrous scaffolds with bioactive collagen molecules," Journal of Biomedical Materials Research Part A, vol. 83, no. 4, pp. 1117-1127, 2007.

[11] S. Torres-Giner, J. V. Gimeno-Alcañiz, M. J. Ocio, and J. M. Lagaron, "Optimization of electrospun polylactide-based ultrathin fibers for osteoconductive bone scaffolds," Journal of Applied Polymer Science, vol. 122, no. 2, pp. 914-925, 2011. 
[12] S. Wang, Y. Zhang, G. Yin, H. Wang, and Z. Dong, "Electrospun polylactide/silk fibroin-gelatin composite tubular scaffolds for small-diameter tissue engineering blood vessels," Journal of Applied Polymer Science, vol. 113, no. 4, pp. 2675-2682, 2009.

[13] G. Yin, Y. Zhang, J. Wu, S. Wang, D. Shi, and Z. Dong, "Study on the Electrospun Poly(lactic acid)/Silk broin-gelatin Composite Nanofibrous Scaffold for Tissue Engineering," Journal of Fiber Bioengineering and Informatics, vol. 2, no. 3, pp. 182-188, 2009.

[14] S.-Y. Gu, Z.-M. Wang, J. Ren, and C.-Y. Zhang, "Electrospinning of gelatin and gelatin/poly(L-lactide) blend and its characteristics for wound dressing," Materials Science and Engineering C: Materials for Biological Applications, vol. 29, no. 6, pp. 18221828, 2009.

[15] H.-W. Kim, H.-S. Yu, and H.-H. Lee, "Nanofibrous matrices of poly(lactic acid) and gelatin polymeric blends for the improvement of cellular responses," Journal of Biomedical Materials Research Part A, vol. 87, no. 1, pp. 25-32, 2008.

[16] W. Y. Ip, "Polylactide membranes and sponges in the treatment of segmental defects in rabbit radii," Injury, vol. 33 , no. 2, pp. 66-70, 2002.

[17] A. Gerber, A. Gerber, and S. Gogolewski, "Reconstruction of large segmental defects in the sheep tibia using polylactide membranes. A clinical and radiographic report," Injury, vol. 33, no. 2, pp. 43-57, 2002.

[18] Z. Gugala and S. Gogolewski, "Healing of critical-size segmental bone defects in the sheep tibiae using bioresorbable polylactide membranes," Injury, vol. 33, no. 2, pp. 71-76, 2002.

[19] L. Zhang and T. J. Webster, "Nanotechnology and nanomaterials: promises for improved tissue regeneration," Nano Today, vol. 4, no. 1, pp. 66-80, 2009.

[20] M. M. Stevens, "Biomaterials for bone tissue engineering," Materials Today, vol. 11, no. 5, pp. 18-25, 2008.

[21] J. M. Holzwarth and P. X. Ma, "Biomimetic nanofibrous scaffolds for bone tissue engineering," Biomaterials, vol. 32, no. 36, pp. 9622-9629, 2011.

[22] W. J. Li, R. Tuli, C. Okafor et al., "A three-dimensional nanofibrous scaffold for cartilage tissue engineering using human mesenchymal stem cells," Biomaterials, vol. 26, no. 6, pp. 599609, 2005.

[23] E. J. Levorson, P. R. Sreerekha, K. P. Chennazhi, F. K. Kasper, S. V. Nair, and A. G. Mikos, "Fabrication and characterization of multiscale electrospun scaffolds for cartilage regeneration," Biomedical Materials, vol. 8, no. 1, Article ID 014103, 2013.

[24] H. J. Shin, C. H. Lee, I. H. Cho et al., "Electrospun PLGA nanofiber scaffolds for articular cartilage reconstruction: mechanical stability, degradation and cellular responses under mechanical stimulation in vitro," Journal of Biomaterials Science, Polymer Edition, vol. 17, no. 1, pp. 103-119, 2006.

[25] Y.-S. Lee and T. L. Arinzeh, "Electrospun nanofibrous materials for neural tissue engineering," Polymer, vol. 3, no. 1, pp. 413-426, 2011.

[26] M. P. Prabhakaran, J. Venugopal, C. K. Chan, and S. Ramakrishna, "Surface modified electrospun nanofibrous scaffolds for nerve tissue engineering," Nanotechnology, vol. 19, no. 45, Article ID 455102, 2008.

[27] F. Yang, R. Murugan, S. Wang, and S. Ramakrishna, "Electrospinning of nano/micro scale poly(L-lactic acid) aligned fibers and their potential in neural tissue engineering," Biomaterials, vol. 26, no. 15, pp. 2603-2610, 2005.

[28] A. J. Salgado, O. P. Coutinho, and R. L. Reis, "Bone tissue engineering: State of the art and future trends," Macromolecular Bioscience, vol. 4, no. 8, pp. 743-765, 2004.
[29] S. Shao, S. Zhou, L. Li et al., "Osteoblast function on electrically conductive electrospun PLA/MWCNTs nanofibers," Biomaterials, vol. 32, no. 11, pp. 2821-2833, 2011.

[30] Y. Matsuda, T. Tsukahara, Y. Usui et al., "DJ-1asapotentialbiomarker for the development of biocompatible multiwalled carbon nanotubes," International Journal of Nanomedicine, Article ID 343747, p. 2689, 2012.

[31] D. Lahiri, F. Rouzaud, S. Namin et al., "Carbon nanotube reinforced polylactide-caprolactone copolymer: mechanical strengthening and interaction with human osteoblasts in vitro," ACS Applied Materials \& Interfaces, vol. 1, no. 11, pp. 2470-2476, 2009.

[32] T. Yang, D. Wu, L. Lu, W. Zhou, and M. Zhang, "Electrospinning of polylactide and its composites with carbon nanotubes," Polymer Composites, vol. 32, no. 8, pp. 1280-1288, 2011.

[33] P. Pötschke, A. R. Bhattacharyya, A. Janke et al., "Melt mixing as method to disperse carbon nanotubes into thermoplastic polymers," Fullerenes, Nanotubes and Carbon Nanostructures, vol. 13, no. supplement 1, pp. 211-224, 2005.

[34] L. Y. Yeo and J. R. Friend, "Electrospinning carbon nanotube polymer composite nanofibers," Journal of Experimental Nanoscience, vol. 1, no. 2, pp. 177-209, 2006.

[35] B. Ercan and T. J. Webster, "The effect of biphasic electrical stimulation on osteoblast function at anodized nanotubular titanium surfaces," Biomaterials, vol. 31, no. 13, pp. 3684-3693, 2010.

[36] A. Fraczek, E. Menaszek, C. Paluszkiewicz, and M. Blazewicz, "Comparative in vivo biocompatibility study of single- and multi-wall carbon nanotubes," Acta Biomaterialia, vol. 4, no. 6, pp. 1593-1602, 2008.

[37] J. Boczkowski and S. Lanone, "Respiratory toxicities of nanomaterials-a focus on carbon nanotubes," Advanced Drug Delivery Reviews, vol. 64, no. 15, pp. 1694-1699, 2012.

[38] A. A. Shvedova, A. Pietroiusti, B. Fadeel, and V. E. Kagan, "Mechanisms of carbon nanotube-induced toxicity: focus on oxidative stress," Toxicology and Applied Pharmacology, vol. 261, no. 2, pp. 121-133, 2012.

[39] A. Fraczek-Szczypta, E. Menaszek, T. B. Syeda et al., "Effect of MWCNT surface and chemical modification on in vitro cellular response," Journal of Nanoparticle Research, vol.14, no. 10, article 1181, 2012.

[40] A. J. Andersen, P. P. Wibroe, and S. M. Moghimi, "Perspectives on carbon nanotube-mediated adverse immune effects," Advanced Drug Delivery Reviews, vol. 64, no. 15, pp. 1700-1705, 2012.

[41] C. L. Ursini, D. Cavallo, A. M. Fresegna et al., "Comparative cyto-genotoxicity assessment of functionalized and pristine multiwalled carbon nanotubes on human lung epithelial cells," Toxicology in Vitro, vol. 26, no. 6, pp. 831-840, 2012.

[42] C. M. Agrawal, J. Best, J. D. Heckman, and B. D. Boyan, "Protein release kinetics of a biodegradable implant for fracture nonunions," Biomaterials, vol. 16, no. 16, pp. 1255-1260, 1995.

[43] J. C. Middleton and A. J. Tipton, "Synthetic biodegradable polymers as orthopedic devices," Biomaterials, vol. 21, no. 23, pp. 2335-2346, 2000.

[44] Y. Dong, S. Liao, M. Ngiam, C. K. Chan, and S. Ramakrishna, "Degradation behaviors of electrospun resorbable polyester nanofibers," Tissue Engineering - Part B: Reviews, vol. 15, no. 3, pp. 333-351, 2009.

[45] R. Scaffaro, F. Lopresti, and L. Botta, "Preparation, characterization and hydrolytic degradation of PLA/PCL co-mingled 
nanofibrous mats prepared via dual-jet electrospinning," European Polymer Journal, vol. 96, pp. 266-277, 2017.

[46] L. Xu, K. Crawford, and C. B. Gorman, "Effects of Temperature and $\mathrm{pH}$ on the Degradation of Poly(lactic acid) Brushes," Macromolecules, vol. 44, no. 12, pp. 4777-4782, 2011.

[47] Y. You, B.-M. Min, S. J. Lee, T. S. Lee, and W. H. Park, "In vitro degradation behavior of electrospun polyglycolide, polylactide, and poly(lactide-co-glycolide)," Journal of Applied Polymer Science, vol. 95, no. 2, pp. 193-200, 2005.

[48] Y. Dong, T. Yong, S. Liao, C. K. Chan, M. M. Stevens, and S. Ramakrishna, "Distinctive degradation behaviors of electrospun polyglycolide, poly(dl-Lactide-co-Glycolide), and poly(lLactide-co-E-Caprolactone) nanofibers cultured with/without porcine smooth muscle cells," Tissue Engineering Part: A, vol. 16, no. 1, pp. 283-298, 2010.

[49] A. Fraczek-Szczypta, E. Menaszek, and S. Blazewicz, "Some observations on carbon nanotubes susceptibility to cell phagocytosis," Journal of Nanomaterials, vol. 2011, Article ID 473516, 8 pages, 2011.

[50] J. Markowski, A. Magiera, M. Lesiak, A. L. Sieron, J. Pilch, and S. Blazewicz, "Preparation and characterization of nanofibrous polymer scaffolds for cartilage tissue engineering," Journal of Nanomaterials, vol. 2015, Article ID 564087, 2015.

[51] A. Socha and M. Blazewicz, "Microscopic studies of polylactide electrospun fibres designed for tissue engineering scaffolds," Engineering of Biomaterials, vol. 115, pp. 12-17, 2012.

[52] D. Battegazzore, S. Bocchini, and A. Frache, "Crystallization kinetics of poly(lactic acid)-talc composites," Express Polymer Letters, vol. 5, no. 10, pp. 849-858, 2011.

[53] S. Soliman, S. Pagliari, A. Rinaldi et al., "Multiscale threedimensional scaffolds for soft tissue engineering via multimodal electrospinning," Acta Biomaterialia, vol. 6, no. 4, pp. 1227-1237, 2010.

[54] X. H. Zong, H. Bien, C.-Y. Chung et al., "Electrospun finetextured scaffolds for heart tissue constructs," Biomaterials, vol. 26, no. 26, pp. 5330-5338, 2005.

[55] K. Fukushima, C. Abbate, D. Tabuani, M. Gennari, and G. Camino, "Biodegradation of poly(lactic acid) and its nanocomposites," Polymer Degradation and Stability, vol. 94, no. 10, pp. 1646-1655, 2009.

[56] M. Glarner and S. Gogolewski, "Degradation and calcification in vitro of new bioresorbable terpolymers of lactides with an improved degradation pattern," Polymer Degradation and Stability, vol. 92, no. 2, pp. 310-316, 2007.

[57] J. Ferdous, V. B. Kolachalama, and T. Shazly, "Impact of polymer structure and composition on fully resorbable endovascular scaffold performance," Acta Biomaterialia, vol. 9, no. 4, pp. 6052-6061, 2013. 


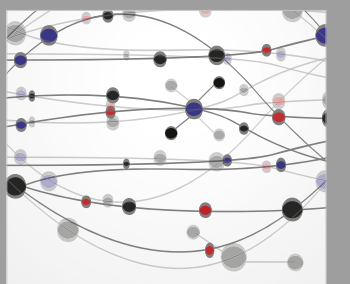

The Scientific World Journal
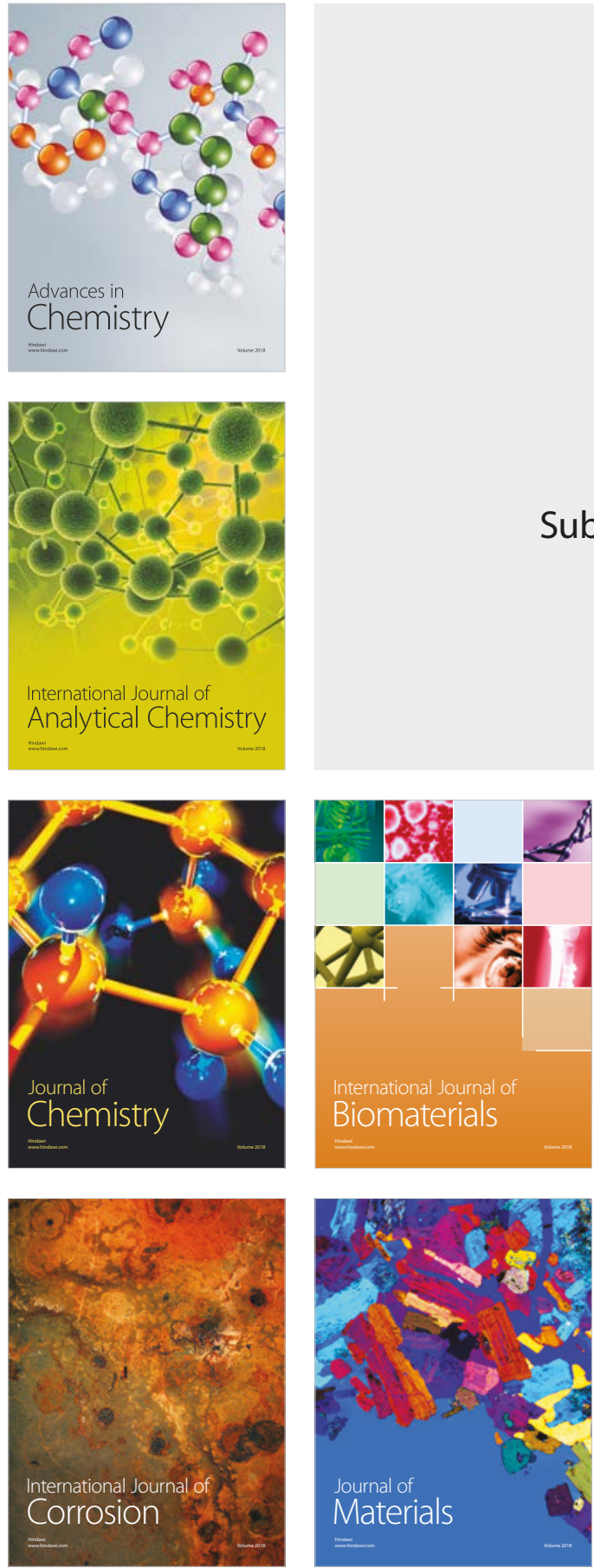

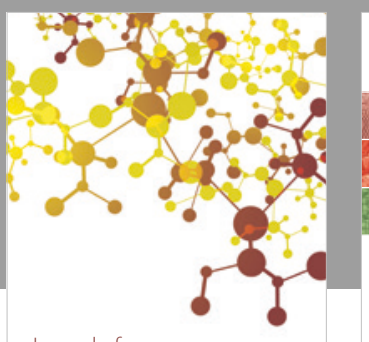

Journal of

Applied Chemistry
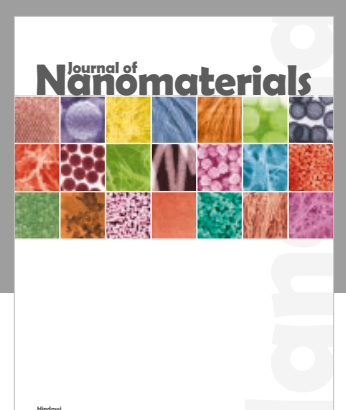

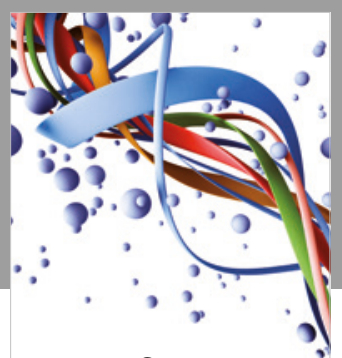

Scientifica

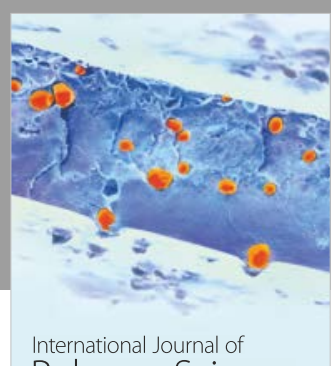

Polymer Science

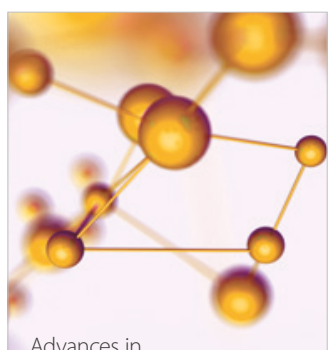

Physical Chemistry
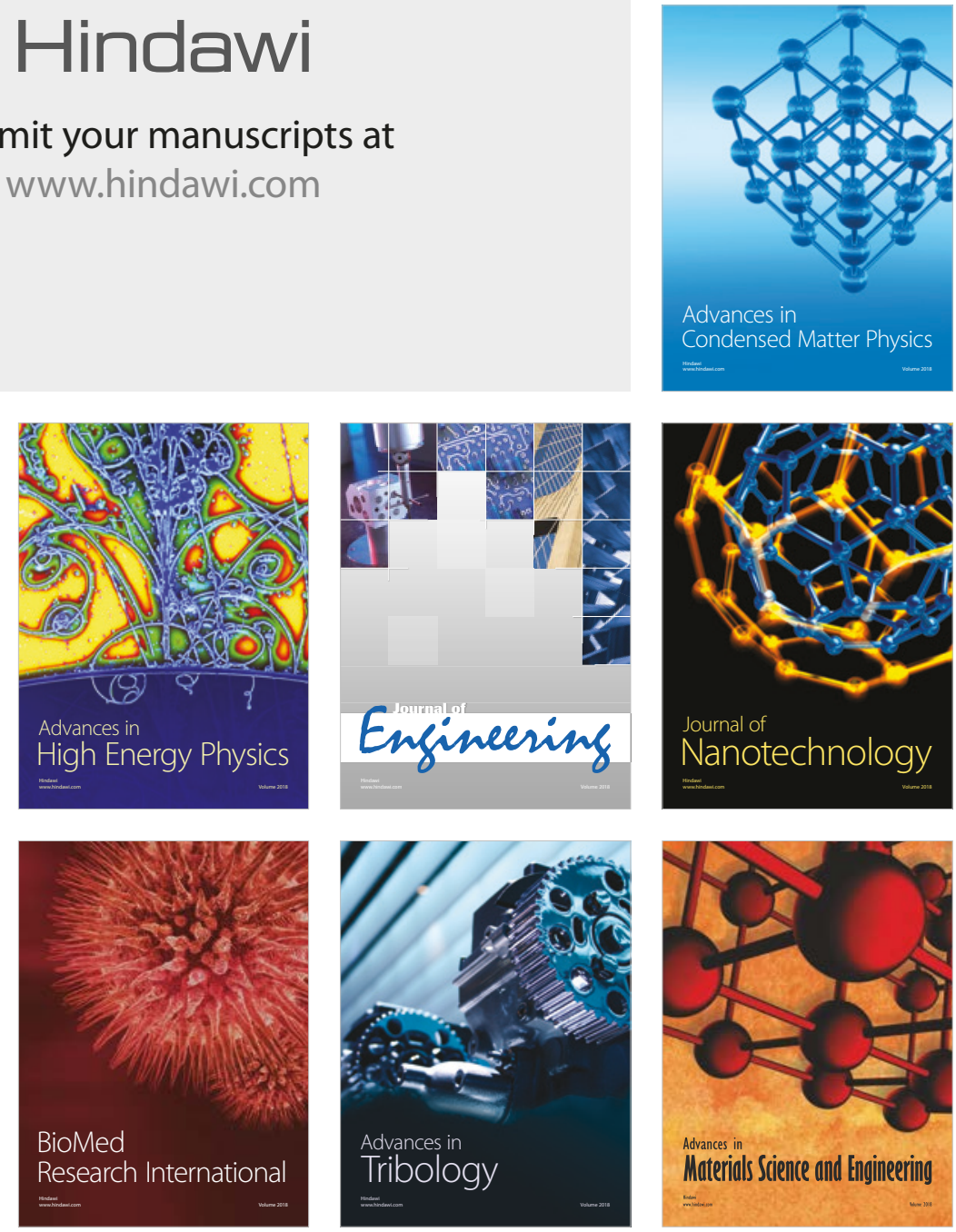